\title{
QUANTUM SUPERGROUPS II. CANONICAL BASIS
}

\author{
SEAN CLARK, DAVID HILL, AND WEIQIANG WANG
}

\begin{abstract}
Following Kashiwara's algebraic approach, we construct crystal bases and canonical bases for quantum supergroups of anisotropic type and for their integrable modules.
\end{abstract}

\section{INTRODUCTION}

The theory of canonical bases for quantum groups and their integrable modules was introduced by Lusztig and subsequently by Kashiwara through a different crystal basis approach (see [Lu1, Lu2, Ka ). Among many applications, the canonical bases have recently played an important role in categorification.

For quantum supergroups, there have been some combinatorial constructions of crystal bases. For quantum osp $(1 \mid 2 n)$, these were constructed for the integrable modules in [Zou] and in Musson and Zou [MZ; also see Jeong [Jeo for a generalization which we will discuss below. For quantum $\mathfrak{g l}(m \mid n)$, crystal bases for the polynomial representations were obtained by Benkart, Kang, and Kashiwara BKK. More recently, crystal bases for a class of infinite-dimensional simple modules of quantum $\mathfrak{o s p}(r \mid 2 n)$ have been constructed in $[\mathrm{Kw}$. However, none of the authors constructed a crystal basis for the negative part of these quantum supergroups. The conventional wisdom among experts seemed to favor the nonexistence of canonical basis (or global crystal basis) for quantum supergroups - until our recent announcement [HW, CW].

The goal of this paper is to systematically develop a theory of canonical bases for half a quantum supergroup and the associated integrable modules for the first time. In the super setting Lusztig's geometric approach is not applicable directly. Instead, we follow Kashiwara's algebraic approach [Ka as a blueprint for this paper.

The class of quantum supergroups $\mathbf{U}$ considered in this paper is of anisotropic type (which means no isotropic odd simple roots) and satisfies an additional barconsistent condition (see [CHW] for a foundation of quantum supergroups of

Received by the editors April 16, 2013 and, in revised form, March 14, 2014.

2010 Mathematics Subject Classification. Primary 17B37.

Key words and phrases. Quantum supergroups, crystal basis, canonical basis.

The first author was partially supported by the Semester Fellowship from Department of Mathematics, University of Virginia (UVA).

The first and third authors gratefully acknowledge the support and stimulating environment at the Institute of Mathematics, Academia Sinica, Taipei, during their visits in Spring 2013.

The third author was partially supported by NSF DMS-1101268 and the UVA Sesqui Fellowship. 
anisotropic type in which we generalized Lusztig [Lu2, Chapter 1]; see also earlier related works [Ya, BKM] $)$. This class of anisotropic type quantum supergroups includes quantum $\mathfrak{o s p}(1 \mid 2 n)$ as the only finite type examples. As noted by two of the authors [HW, it is conceptual to introduce what we call quantum covering groups with an additional formal parameter $\pi$ with $\pi^{2}=1$ in place of a super sign for this class of quantum supergroups, and the quantum covering groups (and, respectively, the quantum supergroups) afford a novel bar involution which sends $q \mapsto \pi q^{-1}$ (and, respectively, by a specialization $\pi \mapsto-1$ ). Our work [HW CW] was motivated by EKL, KKT,Wa and in turn led to new development in categorification of quantum covering and super groups KKO, ELa.

Kashiwara's approach starts with developing a combinatorial theory of crystal basis, which is roughly speaking a basis at $q=0$. An earlier paper of Jeong Jeo] constructs crystal bases for integrable modules $V(\lambda)$ for (a variant of) quantum supergroups of anisotropic type. However, our work differs from loc. cit. in several aspects. First, we deal with the integrable modules $V(\lambda)$ for all dominant integral weights $\lambda \in P^{+}$while Jeong put a restriction on $\lambda$ to a subset of "even" dominant weights (as inherited from [Kac, BKM] ). Some extra generators $J_{\mu}$ of $\mathbf{U}$ introduced in $[\mathrm{CHW}]$ (which was inspired by [CW]) make the constructions of $V(\lambda)$ over $\mathbb{Q}(q)$ for all $\lambda \in P^{+}$possible. Secondly, the odd rank one quantum osp $(1 \mid 2)[\mathrm{CW}]$ admits a 2-dimensional simple module $V(1)$ (here 1 is an "odd" weight unavailable in Jeo]), which plays a basic role in developing the tensor product rule of crystal bases. The tensor product rule in the super setting as developed in [Jeo requires some fixing of super signs, and the proof therein is complicated for lack of this 2-dimensional module. (There is also an earlier version of tensor product rule in [MZ, where the authors had to work with modules over $\mathbb{C}(q)$ instead of $\mathbb{Q}(q)$.)

Here is the layout of the paper, and we mention explicitly when Kashiwara's approach requires more noticeable modifications along the way.

In Section 2, we set up the notations of quantum covering/super groups and provide a quick review of the basics as developed in [CHW]. Two coproducts of $\mathbf{U}$ (differing from each other by some $J_{\mu}$ operators), both corresponding to the one used in Kashiwara's approach, are introduced here. In Section 3, a $(q, \pi)$ boson algebra is introduced in order to formulate the crystal basis for $\mathbf{U}^{-}$, and its basic properties are established. In particular, we introduce a bilinear form (called polarization) on $\mathbf{U}^{-}$and Kashiwara's operators on $\mathbf{U}^{-}$.

In Section 4. we formulate the notion of crystal lattice and crystal basis of integrable modules suitable in the super setting, a variant of which goes back to BKK (for polynomial representations of quantum $\mathfrak{g l}(m \mid n)$ ). A polarization on an integrable module is formulated, and we note an unusual super phenomenon of polarization on a tensor product of two integrable modules. In addition, we establish the tensor product rule of crystal basis in which $\pi$ appears. We formulate the main theorems of crystal bases parallel to Kashiwara's. In Section 5 we adapt Kashiwara's grand loop inductive argument to prove the main theorems of crystal bases. 
In Section 6, we study further properties of polarization. We show that the crystal basis is $\pi$-orthonormal in the sense of Definition 3.14, but in general not orthonormal in the usual sense, with respect to the polarization at $q=0$; in particular, the polarization at $q=0$ is not positive definite. This leads to a key difference in the super setting that neither a crystal lattice nor signed crystal basis in general affords an orthonormality characterization. In the usual quantum group setting such an orthonormality was established by Lusztig and Kashiwara [Lu1, Lu2, Ka], and it readily implies another fundamental fact that the crystal lattice $\mathcal{L}(\infty)$ on $\mathbf{U}^{-}$is preserved by the anti-involution $\varrho$ which fixes each Chevalley generator $F_{i}$. In the super setting, it continues to be true that the crystal lattice $\mathcal{L}(\infty)$ is $\varrho$-stable. The proof of this fact is postponed to [CFLW], as it requires tools somewhat outside the setting of this paper and it follows most readily from the connection between $\mathbf{U}$ and the usual quantum groups developed in loc. cit. (This connection is closely related to a remarkable connection between 2-parameter quantum groups and the usual quantum groups developed by Fan and Li [FLi].)

In Section [7, we establish the existence of canonical bases for $\mathbf{U}^{-}$and all integrable modules $V(\lambda)$ with $\lambda \in P^{+}$.

By $\mathbf{U}$-module in this paper we always mean a $\mathbf{U}$-module with a $\mathbb{Z}_{2}$-grading which is compatible with the action of the superalgebra. By $\mathbf{U}$-homomorphism, we always mean a $\mathbb{Z}_{2}$-graded linear map whose $\mathbb{Z}_{2}$-homogeneous parts supercommute with the U-action.

\section{QuANtum COVERING AND SUPER GROUPS}

2.1. Super generalized Cartan matrix (SGCM). Let $\pi$ and $q$ be formal indeterminants such that $\pi^{2}=1$. For a subring $R$ of the rational function field $\mathbb{Q}(q)$, define a new ring

$$
R^{\pi}=R \otimes_{\mathbb{Z}} \mathbb{Z}[\pi] .
$$

We note the following properties of this ring: $R^{\pi}$ is a subring of $\mathbb{Q}(q)^{\pi} ; x \in R^{\pi}$ is a zero divisor if and only if $x=r(\pi \pm 1)$ for some $r \in R$.

For $n \in \mathbb{Z}$ and $a \in \mathbb{N}$, we define the $(q, \pi)$-integer

$$
[n]=\frac{(\pi q)^{n}-q^{-n}}{\pi q-q^{-1}} \in \mathbb{Z}^{\pi}\left[q, q^{-1}\right]
$$

and then define the corresponding $(q, \pi)$-factorials and $(q, \pi)$-binomial coefficients for $a \in \mathbb{N}$ by

$$
[a]^{!}=\prod_{i=1}^{a}[i], \quad\left[\begin{array}{l}
n \\
a
\end{array}\right]=\frac{\prod_{i=1}^{a}[n+i-a]}{[a]^{!}} .
$$

We adopt the convention that $[0]^{!}=1$. Note that $\left[\begin{array}{l}n \\ a\end{array}\right]=\frac{[n]^{!}}{[a]^{!}[n-a]^{!}}$, for $n \geq a \geq 0$. There is a unique $\mathbb{Q}^{\pi}$-linear map

$$
\psi: \mathbb{Q}(q)^{\pi} \longrightarrow \mathbb{Q}(q)^{\pi}, \quad q \mapsto \pi q^{-1} .
$$

We will also use the notation $\overline{f(q)}:=\psi(f(q))$ and call this map the bar involution. Note that the induced map on $\mathbb{Q}(q)^{\pi} /\langle\pi-1\rangle \cong \mathbb{Q}(q)$ is the usual bar involution. 
Let $I$ be a finite set and fix a partition $I=I_{0} \coprod I_{1}$ such that $I_{1} \neq \emptyset$. We define the parity function $p(\cdot): I \rightarrow \mathbb{Z}_{2}$ via $p(i)=t$ if $i \in I_{t}$.

Assume $|I|=\ell$. We call a matrix $A=\left(a_{i j}\right)_{i, j \in I}$ a super generalized Cartan matrix (SGCM) of anisotropic type if $A$ satisfies the following conditions:

(a) $a_{i i}=2$ for $i \in I$;

(b) $a_{i j} \in-\mathbb{N}$ for $i \neq j \in I$;

(c) $a_{i j}=0$ if and only if $a_{j i}=0$ for $i, j \in I$;

(d) $a_{i j} \in 2 \mathbb{Z}$ if $i \in I_{1}$ and $j \in I$;

(e) there exists an invertible diagonal matrix $D=\operatorname{diag}\left(d_{i}: i \in I\right)$ with $d_{i} \in \mathbb{Z}_{>0}$ and $\operatorname{gcd}\left(d_{i}: i \in I\right)=1$ such that $D A$ is symmetric.

We associate to a SGCM of anisotropic type the following data:

- a finite dimensional $\mathbb{Q}$-vector space $\mathfrak{h}$;

- linearly independent subsets $\left\{\alpha_{i} \in \mathfrak{h}^{*} \mid i \in I\right\}$ and $\left\{\alpha_{i}^{\vee} \in \mathfrak{h} \mid i \in I\right\}$ such that

$$
\left\langle\alpha_{i}^{\vee}, \alpha_{j}\right\rangle=a_{i j}
$$

(Here and below we denote the natural pairing between $\mathfrak{h}$ and $\mathfrak{h}^{*}$ by $\langle\cdot, \cdot\rangle \cdot$ ) - a lattice $P$ in $\mathfrak{h}^{*}$ containing $\alpha_{j}(j \in I)$ such that $\left\langle\alpha_{i}^{\vee}, \lambda\right\rangle \in \mathbb{Z}(i \in I, \lambda \in P)$.

We define the lattice $P^{\vee}:=\{h \in \mathfrak{h} \mid\langle h, P\rangle \subseteq \mathbb{Z}\}$. We have a sublattice $Q:=$ $\bigoplus_{i \in I} \mathbb{Z} \alpha_{i} \subset P$ and a lattice $Q^{\vee}:=\bigoplus_{i \in I} \mathbb{Z} \alpha_{i}^{\vee} \subset P^{\vee}$. Let $Q^{+}=\bigoplus_{I} \mathbb{Z}_{\geq 0} \alpha_{i}$ and $Q^{-}=-Q^{+}$. Denote $P^{+}=\left\{\lambda \in P \mid\left\langle\alpha_{i}^{\vee}, \lambda\right\rangle \in \mathbb{Z}_{\geq 0}, \forall i \in I\right\}$. Fix $\omega_{j} \in P^{+}$for each $j \in I$ such that $\left\langle\alpha_{i}^{\vee}, \omega_{j}\right\rangle=\delta_{i j}$ for $i \in I$.

SGCM's of anisotropic type were first introduced in $\mathrm{Kac}$ though the terminology "anisotropic type" is new (which means that there are no isotropic odd simple roots), and condition (d) above ensures that all the odd simple roots are of type $\mathfrak{o s p}(1 \mid 2)$.

For $i \in I$ set

$$
q_{i}=q^{d_{i}}, \quad \pi_{i}=\pi^{p(i)}, \quad[n]_{i}=\frac{\left(\pi_{i} q_{i}\right)^{n}-q_{i}^{-n}}{\pi_{i} q_{i}-q_{i}^{-1}},
$$

and so on. Throughout this paper, we will always assume that a SGCM is of anisotropic type satisfying the following additional bar-consistent assumption:

(f) $p(i)=d_{i} \bmod 2$.

This assumption immediately implies $\overline{[n]_{i}}=[n]_{i}$, and is indispensable in formulating the bar involution and canonical basis which is the main goal of this paper. We note, however, that this assumption (f) is unnecessary for the definition of the quantum cover groups given in [CHW] (see \$2.2 below) and the $(q, \pi)$-boson algebra introduced in Section 3 .

2.2. Quantum covering group. Let $A$ be a SGCM. The quantum covering group associated to $A$ is defined to be the associative $\mathbb{Q}(q)^{\pi}$-superalgebra $\mathbf{U}_{q, \pi}(A)$ (with 1) generated by

$$
E_{i}, \quad F_{i}, \quad J_{\mu}, \quad K_{\mu} \quad\left(i \in I, \mu \in P^{\vee}\right),
$$


subject to the relations (2.2) $-(2.9)$ below:

$$
\begin{aligned}
K_{0}=1, \quad K_{\mu} K_{\nu}=K_{\mu+\nu}, & \text { for } \mu, \nu \in P^{\vee}, i \in I ; \\
J_{2 \mu}=1, \quad J_{\mu} J_{\nu}=J_{\mu+\nu}, & \text { for } \mu, \nu \in P^{\vee}, i \in I ; \\
J_{\mu} K_{\nu}=K_{\nu} J_{\mu}, & \text { for } \mu, \nu \in P^{\vee} ; \\
K_{\mu} E_{i}=q^{\left\langle\mu, \alpha_{i}\right\rangle} E_{i} K_{\mu}, \quad J_{\mu} E_{i}=\pi^{\left\langle\mu, \alpha_{i}\right\rangle} E_{i} J_{\mu}, & \text { for } i \in I, \mu \in P^{\vee} ; \\
K_{\mu} F_{j}=q^{-\left\langle\mu, \alpha_{j}\right\rangle} F_{j} K_{\mu}, \quad J_{\mu} F_{i}=\pi^{-\left\langle\mu, \alpha_{i}\right\rangle} F_{i} J_{\mu}, & \text { for } i, j \in I, \mu \in P^{\vee} ; \\
\sum_{s+t=1-a_{i j}}(-1)^{s} \pi_{i}^{s p(j)+\left(\begin{array}{c}
s \\
2
\end{array}\right)}\left[\begin{array}{c}
1-a_{i j} \\
s
\end{array}\right]_{i} E_{i}^{t} E_{j} E_{i}^{s}=0, & \text { for } i \neq j \in I ; \\
\sum_{s+t=1-a_{i j}}(-1)^{s} \pi_{i}^{s p(j)+\left(\begin{array}{c}
s \\
2
\end{array}\right)}\left[\begin{array}{c}
1-a_{i j} \\
s
\end{array}\right]_{i} F_{i}^{t} F_{j} F_{i}^{s}=0, & \text { for } i \neq j \in I ; \\
E_{i} F_{j}-\pi^{p(i) p(j)} F_{j} E_{i}=\delta_{i j} \frac{\widetilde{J}_{i} \widetilde{K}_{i}-\widetilde{K}_{-i}}{\pi_{i} q_{i}-q_{i}^{-1},} & \text { for } i, j \in I .
\end{aligned}
$$

In the above, we use the notations $K_{i}:=K_{\alpha_{i}^{\vee}}$ and $J_{i}:=J_{\alpha_{i}^{\vee}}$ for $i \in I$, and $\widetilde{K}_{\nu}=\prod_{i} K_{i}^{d_{i} \nu_{i}}$ and $\widetilde{J}_{\nu}=\prod_{i} J_{i}^{d_{i} \nu_{i}}$ for any $\nu=\sum_{i} \nu_{i} \alpha_{i}^{\vee} \in Q^{\vee}$. In particular,

$$
\widetilde{K}_{i}=K_{i}^{d_{i}}, \quad \widetilde{J}_{i}=J_{i}^{d_{i}} .
$$

The superalgebra structure on $\mathbf{U}_{q, \pi}$ is given by letting $E_{i}, F_{i}$ for $i \in I_{1}$ be odd and the other generators be even. In particular, $\mathbf{U}_{q, \pi}(A)$ inherits a $\mathbb{Z}_{2}$-grading from $I$ :

$$
p\left(E_{i}\right)=p\left(F_{i}\right)=p(i), \quad p\left(K_{\mu}\right)=p\left(J_{\mu}\right)=0, \quad\left(i \in I, \mu \in P^{\vee}\right) .
$$

Remark 2.1. Let $I=\{i\}=I_{1}$ and $Q=P^{\vee}=\mathbb{Z} \alpha_{i}^{\vee}$, and so $A$ is the SGCM associated to the Lie superalgebra $\mathfrak{o s p}(1 \mid 2)$. Then $\mathbf{U}_{q, \pi}(A)$ specialized at $\pi=-1$ is a variant of the quantum osp $(1 \mid 2) U_{0} \oplus U_{1}$ defined in [CW]. That is, $\mathbf{U}_{q, \pi}(A) /\left\langle\pi+1, J_{i}-1\right\rangle$ $\cong U_{0}$ and $\mathbf{U}_{q, \pi} /\left\langle\pi+1, J_{i}+1\right\rangle \cong U_{1}$.

Henceforth, we fix a SGCM $A$ and use the shorthand notation $\mathbf{U}=\mathbf{U}_{q, \pi}(A)$. The algebra $\mathbf{U}$ has a triangular decomposition $\mathbf{U} \cong \mathbf{U}^{-} \otimes \mathbf{U}^{0} \otimes \mathbf{U}^{+}$where $\mathbf{U}^{-}$ (resp. $\mathbf{U}^{+}, \mathbf{U}^{0}$ ) is the subalgebra generated by $\left\{F_{i} \mid i \in I\right\}$ (resp. $\left\{E_{i} \mid i \in I\right\}$, $\left\{K_{\mu}, J_{\mu} \mid \mu \in P^{\vee}\right\}$ ); see [CHW]. For $i \in I$, and $n \geq 1$ define the divided powers $E_{i}^{(n)}=E_{i}^{n} /[n]_{i}^{!}, F_{i}^{(n)}=F_{i}^{n} /[n]_{i}^{!}$. The following fact will be used often later on.

Lemma 2.2. For each $k \in I, \widetilde{J}_{k}$ is central in $\mathbf{U}$.

Proof. It suffices to check that $\widetilde{J}_{k}$ commutes with every $E_{i}$ and $F_{i}$. By (2.5) and (2.6) and using $\widetilde{J}_{i}=J_{i}^{d_{i}}$, this is equivalent to checking that $\pi^{d_{k} a_{k i}}=1$ for all $k, i \in I$, which follows from conditions (d) and (f) in $\$ 2.1$.

2.3. Coproducts. Let $\mathbf{U} \otimes \mathbf{U}$ be the space $\mathbf{U} \otimes_{\mathbb{Q}(q)^{\pi}} \mathbf{U}$ with the usual $\mathbb{Z}_{2^{-}}$-grading. We endow $\mathbf{U} \otimes \mathbf{U}$ with a multiplication given by

$$
(a \otimes b)(c \otimes d)=\pi^{p(b) p(c)}(a c \otimes b d)
$$

for homogeneous $a, b, c, d \in \mathbf{U}$. This makes $\mathbf{U} \otimes \mathbf{U}$ into an associative $\mathbb{Q}(q)^{\pi}$ superalgebra with 1 . 
We have a superalgebra homomorphism $\Delta: \mathbf{U} \longrightarrow \mathbf{U} \otimes \mathbf{U}$ given by

$$
\begin{aligned}
\Delta\left(E_{i}\right) & =E_{i} \otimes \widetilde{K}_{i}^{-1}+\widetilde{J}_{i} \otimes E_{i}, \\
\Delta\left(F_{i}\right) & =F_{i} \otimes 1+\widetilde{K}_{i} \otimes F_{i}, \\
\Delta\left(K_{\mu}\right) & =K_{\mu} \otimes K_{\mu}, \\
\Delta\left(J_{\mu}\right) & =J_{\mu} \otimes J_{\mu},
\end{aligned}
$$

where $i \in I, \mu \in P^{\vee}$. This homomorphism is in fact a coproduct which makes $\mathbf{U}$ a Hopf superalgebra in a suitable sense (a different coproduct was used in [CHW] which is compatible with [Lu2] rather than with [Ka] $)$. We have

$$
\begin{aligned}
& \Delta\left(E_{i}^{(p)}\right)=\sum_{p^{\prime}+p^{\prime \prime}=p} q_{i}^{p^{\prime} p^{\prime \prime}} \widetilde{J}_{i}^{\left(p^{\prime \prime}\right)} E_{i}^{\left(p^{\prime}\right)} \otimes \widetilde{K}_{i}^{-p^{\prime}} E_{i}^{\left(p^{\prime \prime}\right)}, \\
& \Delta\left(F_{i}^{(p)}\right)=\sum_{p^{\prime}+p^{\prime \prime}=p}\left(\pi_{i} q_{i}\right)^{-p^{\prime} p^{\prime \prime}} F_{i}^{\left(p^{\prime}\right)} \widetilde{K}_{i}^{p^{\prime \prime}} \otimes F_{i}^{\left(p^{\prime \prime}\right)} .
\end{aligned}
$$

For $\mathbf{U}$-modules $M$ and $N$, we endow the tensor product $M \otimes N$ with a $\mathbf{U}$-module structure induced by $\Delta$.

There is another coproduct

$$
\Delta^{\prime}: \mathbf{U} \longrightarrow \mathbf{U} \otimes \mathbf{U}
$$

given by

$$
\begin{aligned}
\Delta^{\prime}\left(E_{i}\right) & =E_{i} \otimes \widetilde{K}_{i}^{-1}+1 \otimes E_{i}, \\
\Delta^{\prime}\left(F_{i}\right) & =F_{i} \otimes 1+\widetilde{J}_{i} \widetilde{K}_{i} \otimes F_{i} \\
\Delta^{\prime}\left(K_{\mu}\right) & =K_{\mu} \otimes K_{\mu} \\
\Delta^{\prime}\left(J_{\mu}\right) & =J_{\mu} \otimes J_{\mu} .
\end{aligned}
$$

This induces a second $\mathbf{U}$-module structure on the tensor product of $M$ and $N$, and we denote this $\mathbf{U}$-module by $M \otimes^{\prime} N$.

Remark 2.3. In case $I=I_{0}$, we have $\Delta=\Delta^{\prime}$, and this coproduct coincides with $\Delta_{-}$in [Ka, (1.4.3)] used in the construction of crystal bases therein. In the general super case, there is no compelling reason to choose one of these coproducts over the other. In fact, both $\Delta$ and $\Delta^{\prime}$ will be useful, as we shall see in Lemma 4.9.

2.4. Module categories. The specialization at $\pi=1$ (respectively, at $\pi=-1$ ) of a $\mathbb{Q}(q)^{\pi}$-algebra $R$ is understood as $\mathbb{Q}(q) \otimes_{\mathbb{Q}(q)^{\pi}} R$, where $\mathbb{Q}(q)$ is the $\mathbb{Q}(q)^{\pi}$-module with $\pi$ acting as 1 (respectively, as -1 ). The specialization at $\pi=1$ of the algebra $\mathbf{U}$, denoted by $\mathbf{U}_{+}$, is a variant of this quantum group, with some extra central elements $J_{\mu}$ that act trivially on all representations (see below). The specialization at $\pi=-1$ of $\mathbf{U}$, denoted by $\mathbf{U}_{-}$, is a quantum supergroup, and the extra generators $J_{\mu}$ allow us to formulate integrable modules $V(\lambda)$ for all $\lambda \in P^{+}$, which was not possible before; see CHW.

In the remainder of this paper, by a representation of the algebra $\mathbf{U}$ we mean a $\mathbb{Q}(q)^{\pi}$-module on which $\mathbf{U}$ acts. Note we have a direct sum decomposition of 
the $\mathbb{Q}(q)^{\pi}$-module $\mathbb{Q}(q)^{\pi} \cong \mathbb{Q}(q)^{\pi} /\langle\pi-1\rangle \oplus \mathbb{Q}(q)^{\pi} /\langle\pi+1\rangle$, where $\pi$ acts as \pm 1 on $\mathbb{Q}(q)^{\pi} /\langle\pi \mp 1\rangle \cong \mathbb{Q}(q)$.

We define the category $\mathcal{C}$ (of weight $\mathbf{U}$-modules) as follows. An object of $\mathcal{C}$ is a $\mathbb{Z}_{2}$-graded $\mathbf{U}$-module $M=M_{\overline{0}} \oplus M_{\overline{1}}$, compatible with the $\mathbb{Z}_{2}$-grading on $\mathbf{U}$, with a given weight space decomposition

$$
M=\bigoplus_{\lambda \in X} M^{\lambda}, \quad M^{\lambda}=\left\{m \in M \mid K_{\mu} m=q^{\langle\mu, \lambda\rangle} m, J_{\mu} m=\pi^{\langle\mu, \lambda\rangle} m, \forall \mu \in P^{\vee}\right\},
$$

such that $M^{\lambda}=M_{\overline{0}}^{\lambda} \oplus M_{\overline{1}}^{\lambda}$ where $M_{\overline{0}}^{\lambda}=M^{\lambda} \cap M_{\overline{0}}$ and $M_{\overline{1}}^{\lambda}=M^{\lambda} \cap M_{\overline{1}}$. The $\mathbb{Z}_{2^{-}}$ graded structure is only particularly relevant to tensor products, and will generally be suppressed when irrelevant. We have the following $\mathbb{Q}(q)^{\pi}$-module decomposition for each weight space: $M^{\lambda} \cong M^{\lambda} /\langle\pi-1\rangle \oplus M^{\lambda} /\langle\pi+1\rangle$; accordingly, we have $M \cong M_{+} \oplus M_{-}$as $\mathbf{U}$-modules, where $M_{ \pm}:=\bigoplus_{\lambda \in X} M^{\lambda} /\langle\pi \mp 1\rangle$ is an U-module on which $\pi$ acts as \pm 1 , i.e., a $\left.\mathbf{U}\right|_{\pi= \pm 1}$-module. Hence the category $\mathcal{C}$ decomposes into a direct sum $\mathcal{C}=\mathcal{C}_{+} \oplus \mathcal{C}_{-}$, where $\mathcal{C}_{ \pm}$can be identified with categories of weight modules over the specializations $\mathbf{U}_{ \pm}$.

The BGG category $\mathcal{O}$ and the category $\mathcal{O}^{\text {int }}$ of integrable modules can be defined as usual (cf. [CHW, $\S 2.5$ and $\S 2.6]$ ). The decomposition $\mathcal{C}=\mathcal{C}_{+} \oplus \mathcal{C}_{-}$gives rise to a similar decomposition $\mathcal{O}^{\text {int }}=\mathcal{O}_{+}^{\text {int }} \oplus \mathcal{O}_{-}^{\text {int }}$ thanks to $\mathcal{O}^{\text {int }} \subset \mathcal{O} \subset \mathcal{C}$.

Let $I(\lambda)$ be the left ideal of $\mathbf{U}$ generated by $\left\{E_{i}, K_{\mu}-q^{\langle\mu, \lambda\rangle}, J_{\mu}-\pi_{i}^{\langle\mu, \lambda\rangle} \mid \mu \in P^{\vee}\right\}$, for each $\lambda \in P$, and define the Verma module associated to $\lambda$ to be $M(\lambda):=\mathbf{U} / I(\lambda)$. We denote the image of $1 \in \mathbf{U}$ in this quotient by $v_{\lambda}^{+}$. Then we have $M(\lambda)=$ $M(\lambda)_{+} \oplus M(\lambda)_{-}$and each $M(\lambda)_{ \pm}$has a unique quotient $\mathbf{U}$-module $V(\lambda)_{ \pm}$. Note that $V(\lambda)_{ \pm}^{\lambda}=\mathbb{Q}(q) v_{\lambda}^{+}$. We emphasize that in our definition, $p\left(v_{\lambda}^{+}\right)=0$. The following was proved in [CHW].

Proposition 2.4 ([CHW, Theorem 3.3.2, Corollary 3.3.3]). The category $\mathcal{O}^{\text {int }}$ is semisimple; $\left\{V(\lambda)_{ \pm} \mid \lambda \in P^{+}\right\}$forms a set of pairwise nonisomorphic simple modules in $\mathcal{O}^{\text {int }}$.

For $M \in \mathcal{C}$ and $m \in M_{\mu}$, we write $|m|=\mu$ and call this the weight grading. In particular, the elements of $\mathbf{U}$ have a weight grading given by $\left|K_{\mu}\right|=\left|J_{\mu}\right|=0$, $\left|E_{i}\right|=\alpha_{i},\left|F_{i}\right|=-\alpha_{i}\left(i \in I, \mu \in P^{\vee}\right)$.

Remark 2.5. The characters of $V(\lambda)_{+}$and $V(\lambda)_{-}$coincide for all $\lambda \in P^{+}$. (This was stated in [CHW], and a proof is given in [KKO] and [CFLW].) Thus, $V(\lambda):=$ $M(\lambda) /\left\langle F_{i}^{\left\langle\alpha_{i}^{\vee}, \lambda\right\rangle+1} v_{\lambda}^{+} \mid i \in I\right\rangle$ is free as a $\mathbb{Q}(q)^{\pi}$-module, and $V(\lambda) \cong V(\lambda)_{+} \oplus V(\lambda)_{-}$.

\section{The $(q, \pi)$-BOSON SUPERALGEBRA}

In this section, we formulate a $(q, \pi)$-version of the $q$-boson algebra as found in Kashiwara [Ka, §3]. Note that when $\pi$ is specialized to 1 , this is just the $q$-boson algebra therein.

3.1. The algebra $\mathbf{B}^{\bullet}$. Let $\mathbf{B}^{\bullet}=\mathbf{B}_{q, \pi}^{\bullet}$ be the $\mathbb{Q}(q)^{\pi}$-superalgebra generated by odd elements $e, f$ subject to the relation

$$
e f=\pi q^{-2} f e+1
$$


We set $f^{(n)}=f^{n} /[n]^{!}$.

One checks that

$$
e^{n} f^{(m)}=\sum_{t \geq 0}(\pi q)^{\left(\begin{array}{c}
t+1 \\
2
\end{array}\right)-n m} q^{-(n-t)(m-t)}\left[\begin{array}{l}
n \\
t
\end{array}\right] f^{(m-t)} e^{n-t} .
$$

The following properties may be directly verified.

Lemma 3.1. Let $M$ be $a \mathbf{B}^{\bullet}$-module which is locally finite for $e$.

(i) $P=\sum_{n \geq 0}(-1)^{n} q^{-\left(\begin{array}{c}n \\ 2\end{array}\right)} f^{(n)} e^{n}$ defines an endomorphism of $M$ satisfying

$$
e P=P f=0 \text { and } \sum_{t \geq 0}(\pi q)^{\left(\begin{array}{l}
t \\
2
\end{array}\right)} f^{(t)} P e^{t}=1 .
$$

(ii) Let $m \in M$. Then any $u \in M$ has a unique decomposition $u=\sum_{n \geq 0} f^{(n)} u_{n}$

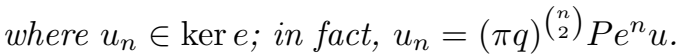

(iii) $M=\operatorname{im} f \oplus \operatorname{ker} e$. Moreover, $P: M \rightarrow M$ is the projection map onto ker $e$ along this direct sum decomposition.

3.2. The algebra B. Define the algebra $\mathbf{B}^{\prime}$ to be the $\mathbb{Q}(q)^{\pi}$-algebra generated by the elements $\left\{e_{i}, f_{i} \mid i \in I\right\}$ subject to the relations

$$
e_{i} f_{j}=\pi^{p(i) p(j)} q_{i}^{-a_{i j}} f_{j} e_{i}+\delta_{i j}, \quad \text { for all } i, j \in I .
$$

Then $\mathbf{B}^{\prime}$ is naturally a superalgebra with parity on generators given by $p\left(e_{i}\right)=$ $p\left(f_{i}\right)=p(i)$ for $i \in I$. Set $f_{i}^{(n)}=f_{i}^{n} /[n]_{i}^{!}$. The superalgebra $\mathbf{B}$ by definition has the same generators as $\mathbf{B}^{\prime}$ subject to the relation (3.3) and the additional $(q, \pi)$-Serre relations (3.4):

$$
\begin{aligned}
& \sum_{t=0}^{b_{i j}}(-1)^{t} \pi_{i}^{\left(\begin{array}{c}
t \\
2
\end{array}\right)+t p(j)}\left[\begin{array}{c}
b_{i j} \\
t
\end{array}\right]_{i} e_{i}^{b_{i j}-t} e_{j} e_{i}^{t}=0, \\
& \sum_{t=0}^{b_{i j}}(-1)^{t} \pi_{i}^{\left(\begin{array}{c}
t \\
2
\end{array}\right)+t p(j)}\left[\begin{array}{c}
b_{i j} \\
t
\end{array}\right]_{i} f_{i}^{b_{i j}-t} f_{j} f_{i}^{t}=0,
\end{aligned}
$$

where we denote

$$
b_{i j}=1-a_{i j}
$$

Let

$$
S_{i j}=\sum_{t=0}^{b_{i j}}(-1)^{t} \pi_{i}^{\left(\begin{array}{l}
t \\
2
\end{array}\right)+t p(j)}\left[\begin{array}{c}
b_{i j} \\
t
\end{array}\right] e_{i}^{b_{i j}-t} e_{j} e_{i}^{t} \in \mathbf{B}^{\prime} .
$$

Lemma 3.2. The following holds in $\mathbf{B}^{\prime}$ for all $i, j, k \in I$ with $i \neq j$ :

$$
S_{i j} f_{k}=\pi_{k}^{b_{i j} p(i)+p(j)} q_{k}^{-\left\langle\alpha_{k}^{\vee}, b_{i j} \alpha_{i}+\alpha_{j}\right\rangle} f_{k} S_{i j}
$$

Proof. Let $C_{i j}^{k}=S_{i j} f_{k}-\pi_{k}^{b_{i j} p(i)+p(j)} q_{k}^{-\left\langle\alpha_{k}^{\vee}, b_{i j} \alpha_{i}+\alpha_{j}\right\rangle} f_{k} S_{i j}$.

If $k \neq i, j$, then it is apparent that $C_{i j}^{k}=0$ from the defining relations. 
When $k=j$, then we have

$$
C_{i j}^{j}=e_{i}^{b_{i j}} \sum_{t=0}^{b_{i j}}(-1)^{t} q_{i}^{-t a_{i j}} \pi_{i}^{\left(\begin{array}{c}
t \\
2
\end{array}\right)}\left[\begin{array}{c}
b_{i j} \\
t
\end{array}\right]_{i}=0,
$$

by using $1-a_{i j}=b_{i j}$ and the identity $\sum_{t=0}^{n}(-1)^{t} q_{i}^{t(n-1)} \pi_{i}^{\left(\begin{array}{c}t \\ 2\end{array}\right)}\left[\begin{array}{l}n \\ t\end{array}\right]_{i}=0$.

Finally, if $k=i$, then we have

$C_{i j}^{i}=\sum_{t=0}^{b_{i j}-1}(-1)^{t} q_{i}^{-t} e_{i}^{b_{i j}-t-1} e_{j} e_{i}^{t} \pi_{i}^{\left(\begin{array}{l}t \\ 2\end{array}\right)+(t+1) p(j)}\left(\pi_{i}^{a_{i j}}\left[\begin{array}{c}b_{i j} \\ t\end{array}\right]_{i}\left[b_{i j}-t\right]_{i}-\left[\begin{array}{c}b_{i j} \\ t+1\end{array}\right]_{i}[t+1]_{i}\right)=0$, by noting $\left[\begin{array}{l}n \\ t\end{array}\right][n-t]=\left[\begin{array}{c}n \\ t+1\end{array}\right][t+1]$ and $a_{i j} p(i) \in 2 \mathbb{Z}$; see condition $(\mathrm{d})$ in $\$ 2.2$, The lemma is proved.

Remark 3.3. A multi-parameter version of the quantum boson algebra can also be found in $\mathrm{KKO}$.

3.3. B-modules. For $\mathbb{Z}_{2}$-homogeneous elements $x, y \in \mathbf{U}$, we write the supercommutator as $[x, y]:=x y-\pi^{p(x) p(y)} y x$. For $i \in I$, define $E_{i}^{\prime}$ and $E_{i}^{\prime \prime}$ in $\operatorname{End}\left(\mathbf{U}^{-}\right)$ by

$$
\left[E_{i}, y\right]=\frac{\widetilde{J}_{i} \widetilde{K}_{i} E_{i}^{\prime \prime}(y)-\widetilde{K}_{i}^{-1} E_{i}^{\prime}(y)}{\pi_{i} q_{i}-q_{i}^{-1}}, \quad \text { for } y \in \mathbf{U}^{-} .
$$

The existence and uniqueness of such linear operators $E_{i}^{\prime}$ and $E_{i}^{\prime \prime}$ is proved easily (actually it can be read off from the proof Lemma 3.4 below).

Lemma 3.4. For $y \in \mathbf{U}^{-}$, we have

$$
\begin{aligned}
& E_{i}^{\prime}\left(F_{j} y\right)=\pi_{i}^{p(j)} q_{i}^{-\left\langle\alpha_{i}^{\vee}, \alpha_{j}\right\rangle} F_{j} E_{i}^{\prime}(y)+\delta_{i j} y, \\
& E_{i}^{\prime \prime}\left(F_{j} y\right)=\pi_{i}^{p(j)} q_{i}^{\left\langle\alpha_{i}^{\vee}, \alpha_{j}\right\rangle} F_{j} E_{i}^{\prime \prime}(y)+\delta_{i j} y .
\end{aligned}
$$

Proof. First we have

$$
\left[E_{i}, F_{j} y\right]=\pi_{i}^{p(j)} F_{j}\left[E_{i}, y\right]+\delta_{i j} \frac{\widetilde{J}_{i} \widetilde{K}_{i}-\widetilde{K}_{i}^{-1}}{\pi_{i} q_{i}-q_{i}^{-1}} y .
$$

It follows by definition that

$$
\begin{aligned}
{\left[E_{i}, F_{j} y\right] } & =\frac{\widetilde{J}_{i} \widetilde{K}_{i} E_{i}^{\prime \prime}\left(F_{j} y\right)-\widetilde{K}_{i}^{-1} E_{i}^{\prime}\left(F_{j} y\right)}{\pi_{i} q_{i}-q_{i}^{-1}}, \\
\pi_{i}^{p(j)} F_{j}\left[E_{i}, y\right] & +\delta_{i j} \frac{\widetilde{J}_{i} \widetilde{K}_{i}-\widetilde{K}_{i}^{-1}}{\pi_{i} q_{i}-q_{i}^{-1}} y \\
& =\frac{\pi_{i}^{p(j)} F_{j}\left(\widetilde{J}_{i} \widetilde{K}_{i} E_{i}^{\prime \prime}(y)-\widetilde{K}_{i}^{-1} E_{i}^{\prime}(y)\right)}{\pi_{i} q_{i}-q_{i}^{-1}}+\delta_{i j} \frac{\widetilde{J}_{i} \widetilde{K}_{i} y-\widetilde{K}_{i}^{-1} y}{\pi_{i} q_{i}-q_{i}^{-1}} .
\end{aligned}
$$

Now the lemma follows by a comparison of the right-hand sides of the two equations above using the commutation relation (2.6) and noting $\widetilde{J}_{i}$ is central.

Proposition 3.5. We have $E_{i}^{\prime} E_{j}^{\prime \prime}=\pi^{p(i) p(j)} q_{j}^{\left\langle\alpha_{j}^{\vee}, \alpha_{i}\right\rangle} E_{j}^{\prime \prime} E_{i}^{\prime}$, for $i, j \in I$. 
Proof. Let $\nu \in Q^{-}$and recall that for $\mu=\sum_{i} a_{i} \alpha_{i} \in Q$ we define its height as ht $\mu=\sum_{i} a_{i}$. Let $y \in U_{\nu}^{-}$.

If ht $(-\nu) \leq 1$, then $E_{i}^{\prime} E_{j}^{\prime \prime}(y)=0=E_{j}^{\prime \prime} E_{i}^{\prime}(y)$. Otherwise, we may assume $y=F_{k} y^{\prime}$ for some $k \in I$ and $y^{\prime} \in U^{-}$with ht $y^{\prime}<$ ht $y$. Then

$$
\begin{aligned}
E_{i}^{\prime} E_{j}^{\prime \prime}(y) & =E_{i}^{\prime}\left(\pi_{j}^{p(k)} q_{j}^{\left\langle\alpha_{j}^{\vee}, \alpha_{k}\right\rangle} F_{k} E_{j}^{\prime \prime}\left(y^{\prime}\right)+\delta_{j k} y^{\prime}\right) \\
& =f(i, j, k) F_{k} E_{i}^{\prime} E_{j}^{\prime \prime}\left(y^{\prime}\right)+\pi_{j}^{p(k)} q_{j}^{\left\langle\alpha_{j}^{\vee}, \alpha_{k}\right\rangle} \delta_{i k} E_{j}^{\prime \prime}\left(y^{\prime}\right)+\delta_{j k} E_{i}^{\prime}\left(y^{\prime}\right) \\
& =f(i, j, k) F_{k} E_{i}^{\prime} E_{j}^{\prime \prime}\left(y^{\prime}\right)+\pi^{p(i) p(j)} q^{d_{j}\left\langle\alpha_{j}^{\vee}, \alpha_{i}\right\rangle} \delta_{i k} E_{j}^{\prime \prime}\left(y^{\prime}\right)+\delta_{j k} E_{i}^{\prime}\left(y^{\prime}\right)
\end{aligned}
$$

and, similarly,

$$
E_{j}^{\prime \prime} E_{i}^{\prime}(y)=f(i, j, k) F_{k} E_{j}^{\prime \prime} E_{i}^{\prime}\left(y^{\prime}\right)+\pi^{p(i) p(j)} q^{-d_{i}\left\langle\alpha_{i}^{\vee}, \alpha_{j}\right\rangle} \delta_{j k} E_{i}^{\prime}\left(y^{\prime}\right)+\delta_{i k} E_{j}^{\prime \prime}\left(y^{\prime}\right),
$$

where we have denoted $f(i, j, k)=\pi^{(p(i)+p(j)) p(k)} q_{j}^{\left\langle\alpha_{j}^{\vee}, \alpha_{k}\right\rangle} q^{-d_{i}\left\langle\alpha_{i}^{\vee}, \alpha_{k}\right\rangle+d_{j}\left\langle\alpha_{j}^{\vee}, \alpha_{k}\right\rangle}$.

Note that $d_{j}\left\langle\alpha_{j}^{\vee}, \alpha_{i}\right\rangle=d_{i}\left\langle\alpha_{i}^{\vee}, \alpha_{j}\right\rangle$ and by induction $E_{i}^{\prime} E_{j}^{\prime \prime}\left(y^{\prime}\right)=\pi^{p(i) p(j)} q_{j}^{\left\langle\alpha_{j}^{\vee}, \alpha_{i}\right\rangle}$ $E_{j}^{\prime \prime} E_{i}^{\prime}\left(y^{\prime}\right)$. Therefore, we have $E_{i}^{\prime} E_{j}^{\prime \prime}(y)=\pi^{p(i) p(j)} q_{j}^{\left\langle\alpha_{j}^{\vee}, \alpha_{i}\right\rangle} E_{j}^{\prime \prime} E_{i}^{\prime}(y)$.

From this we derive the following (see [CHW, Lemma 1.3.15] for an equivalent version, and the equivalence can be read off from (6.6) below).

Corollary 3.6. For $x \in \mathbf{U}^{-}$, if $E_{i}^{\prime}(x)=0$ for all $i \in I$, then $x \in \mathbb{Q}(q)^{\pi}$.

Proof. The proof proceeds as in [Ka, Lemma 3.4.7].

Lemma 3.7. Let $i \in I$ and $u \in \mathbf{U}_{\zeta}^{-}$such that $E_{i}^{\prime}(u)=0$. Then for any $\mathbf{U}$-module $M$ and $m \in M_{\lambda}$ such that $e_{i} m=0$, we have

$$
\widetilde{K}_{i}^{n} E_{i}^{n} u m=\pi_{i}^{n\left\langle\alpha_{i}^{\vee}, \lambda\right\rangle} \frac{q_{i}^{n\left(2\left\langle\alpha_{i}^{\vee}, \lambda+\zeta\right\rangle+3 n+1\right)}}{\left(\pi_{i} q_{i}-q_{i}^{-1}\right)^{n}}\left(E_{i}^{\prime \prime n} u\right) m .
$$

Proof. This lemma has essentially the same proof as [Ka, Lemma 3.4.6]. The power of $\pi$ comes from the central element $\widetilde{J}_{i}$.

Our interest in the superalgebra $\mathbf{B}$ comes from the following result.

Proposition 3.8. $\mathbf{U}^{-}$is a $\mathbf{B}^{\prime}$-module as well as a $\mathbf{B}$-module, where $f_{i}$ acts as multiplication by $F_{i}$ and $e_{i}$ acts by the map $E_{i}^{\prime}$ for all $i \in I$.

Proof. By Lemma 3.4, $\mathbf{U}^{-}$is a $\mathbf{B}^{\prime}$-module. Recall the Serre elements $S_{i j} \in \mathbf{B}^{\prime}$ from (3.5) and denote the $f$-counterparts by

$$
S_{i j}^{\prime}=\sum_{t=0}^{b_{i j}}(-1)^{t} \pi_{i}^{\left(\begin{array}{c}
t \\
2
\end{array}\right)+t p(j)}\left[\begin{array}{c}
b_{i j} \\
t
\end{array}\right] f_{i}^{b_{i j}-t} f_{j} f_{i}^{t} \in \mathbf{B}^{\prime} .
$$

To show that $\mathbf{U}^{-}$is a $\mathbf{B}$-module, it suffices to show that $S_{i j}$ and $S_{i j}^{\prime}$ act as zero on any $y \in \mathbf{U}^{-}$. By the definition of the action and the Serre relations in $\mathbf{U}$,

$$
S_{i j}^{\prime} y=\left(\sum_{t=0}^{b_{i j}}(-1)^{t} \pi_{i}^{\left(\begin{array}{c}
t \\
2
\end{array}\right)+t p(j)}\left[\begin{array}{c}
b_{i j} \\
t
\end{array}\right] F_{i}^{b_{i j}-t} F_{j} F_{i}^{t}\right) y=0 .
$$


For $S_{i j}$, we may assume that $y$ is a monomial in the generators $F_{i}$ for $i \in I$, so $y=m(f) 1$ where $m(f) \in \mathbf{B}^{\prime}$ is a monomial in the $f_{i}$ for $i \in I$. By repeated application of Lemma 3.2. $S_{i, j} m(f)=c m(f) S_{i j}$ for some scalar $c \in \mathbb{Q}(q)^{\pi}$. Since $E_{k}^{\prime}(1)=0$ for all $k \in I, S_{i j} 1=0$ whence $S_{i j} y=0$.

Corollary 3.9. As $\mathbf{B}$-modules, $\mathbf{U}^{-} \cong \mathbf{B} / \sum_{i} \mathbf{B} e_{i}$.

Proof. The final remark in the proof of Proposition 3.8 shows that there is a $\mathbf{B}$ module homomorphism $\mathbf{B} / \sum_{i} \mathbf{B} e_{i} \rightarrow \mathbf{U}^{-}$. On the other hand, the $f_{i}$ generate a subalgebra of $\mathbf{B}$ isomorphic to $\mathbf{U}^{-}$, so this map must be an isomorphism.

\subsection{Polarization on $\mathbf{U}^{-}$.}

Proposition 3.10. There is a unique bilinear form $(\cdot, \cdot)$ on $\mathbf{U}^{-}$satisfying

$$
(1,1)=1, \quad\left(f_{i} y, z\right)=\left(y, e_{i} z\right) \quad \forall y, z \in \mathbf{U}^{-}, i \in I .
$$

Moreover, this bilinear form is symmetric.

Proof. First note that there is a unique linear map $a: \mathbf{B} \rightarrow \mathbf{B}$ with $a\left(e_{i}\right)=f_{i}$ and $a\left(f_{i}\right)=e_{i}$, and $a(x y)=a(y) a(x)$ for $x, y \in \mathbf{B}$. Using this, $\left(\mathbf{U}^{-}\right)^{*}$ becomes a B-module via $(p \cdot \phi)(y)=\phi(a(p) \cdot y)$ for $p \in \mathbf{B}, y \in \mathbf{U}^{-}$and $\phi \in\left(\mathbf{U}^{-}\right)^{*}$.

Let $\phi_{0} \in\left(\mathbf{U}^{-}\right)^{*}$ be defined by $\phi_{0}(1)=1$ and $\phi_{0}\left(\sum_{i} f_{i} \mathbf{U}^{-}\right)=0$. Note that $e_{i} \phi_{0}(x)=\phi_{0}\left(f_{i} x\right)=0$ for all $x \in \mathbf{U}^{-}, i \in I$. Therefore, there is a $\mathbf{B}$-homomorphism $\Psi: \mathbf{U}^{-} \rightarrow\left(\mathbf{U}^{-}\right)^{*}$ factoring through the map $\mathbf{B} / \sum \mathbf{B} e_{i} \rightarrow\left(\mathbf{U}^{-}\right)^{*}$; in particular, $1 \mapsto \phi_{0}$.

Define $(\cdot, \cdot)$ on $\mathbf{U}^{-}$by $(y, z)=\Psi(y)(z)$. Then by construction, $(1,1)=\phi_{0}(1)=1$ and $\left(f_{i} y, z\right)=f_{i} \Psi(y)(z)=\Psi(y)\left(e_{i} z\right)=\left(y, e_{i} z\right)$. Clearly, these properties completely determine the bilinear form. Then since the form $(\cdot, \cdot)^{\prime}$ defined by $(y, z)^{\prime}=$ $\Psi(z)(y)$ satisfies the same properties, the symmetry follows by the uniqueness of such a bilinear form.

Corollary 3.11. The bilinear form $(\cdot, \cdot)$ on $\mathbf{U}^{-}$is nondegenerate; moreover, $\left(\mathbf{U}_{\nu}^{-}, \mathbf{U}_{\mu}^{-}\right)=0$ if $\nu \neq \mu$.

Proof. The weight claim follows from the definition of the bilinear form and may be shown by induction on the height of weights. Nondegeneracy of the bilinear form may be shown also by induction on height with a crucial observation as follows: if $0 \neq y \in \mathbf{U}_{\nu}^{-}$with $\nu \neq 0$ such that $\left(y, \mathbf{U}_{\nu}^{-}\right)=0$, then $\left(e_{i} y, \mathbf{U}_{\nu+i}^{-}\right)=0$ for all $i \in I$, whence $e_{i} y=0$ for all $i \in I$. But then by Corollary $3.6 \nu=0$, and hence we have a contradiction.

Note that $\mathbf{U}^{-} \cong \mathbf{U}_{+}^{-} \oplus \mathbf{U}_{-}^{-}$as $\mathbf{B}$-modules, where $\mathbf{U}_{ \pm}^{-}:=\mathbf{U}^{-} /\langle\pi \pm 1\rangle$. The bilinear form $(\cdot, \cdot)$ on $\mathbf{U}^{-}$restricts to bilinear forms on $\mathbf{U}_{ \pm}^{-}$, still denoted by $(\cdot, \cdot)$. The bilinear form $(\cdot, \cdot)$ will be referred to as the polarization on $\mathbf{U}^{-}, \mathbf{U}_{+}^{-}$or $\mathbf{U}_{-}^{-}$. Corollary [3.6] implies the following.

Corollary 3.12. The $\mathbf{B}$-modules $\mathbf{U}_{\diamond}^{-}$, for $\diamond \in\{+,-\}$, are simple. 
3.5. Crystal basis of $\mathbf{U}^{-}$. We define a category $\mathcal{P}$ as follows. The objects of $\mathcal{P}$ are B-modules $M$ such that for any $m \in M$, there exists an $t \in \mathbb{N}$ such that for any $i_{1}, \ldots, i_{t} \in I, e_{i_{1}} \ldots e_{i_{t}} m=0$. The homomorphisms are $\mathbf{B}$-module homomorphisms. Then we have $\mathbf{U}_{\diamond}^{-} \in \mathcal{P}$, for $\diamond \in\{\emptyset,+,-\}$, where by convention we drop the subscript $\emptyset$ in case of $\diamond=\emptyset$. In fact, $\mathbf{U}_{\diamond}^{-}$, for $\diamond \in\{+,-\}$, are the only simple modules up to isomorphism and $\mathcal{P}$ is semisimple.

Lemma 3.13. Let $M \in \mathcal{P}$. For each $i \in I$, every $m \in M$ has a unique expression of the form

$$
m=\sum_{t \geq 0} f_{i}^{(t)} m_{t}
$$

where $m_{t} \in \operatorname{ker} e_{i}$ and $m_{t}$ is nonzero for finitely many $t$.

Proof. By the definition of $\mathcal{P}$, each $e_{i}$ is locally finite on $M$. Note that $e_{i}$ and $f_{i}$ generate a subalgebra of $\mathbf{B}$ isomorphic to $\mathbf{B}_{q_{i}, \pi_{i}}^{\bullet}$ and so Lemma 3.1(ii) finishes the proof.

Let $i \in I$. Let $M \in \mathcal{P}$ and $m \in M$ such that $m=\sum_{t} f_{i}^{(t)} m_{t}$ with $m_{t} \in \operatorname{ker} e_{i}$. We define the Kashiwara operators

$$
\tilde{e}_{i} m=\sum_{t} f_{i}^{(t-1)} m_{t} \quad \text { and } \tilde{f}_{i} m=\sum_{t} f_{i}^{(t+1)} m_{t} .
$$

Note that these operators (super)commute with B-module homomorphisms.

The action of $\mathbf{B}$ on $\mathbf{U}_{+}^{-}=\mathbf{U}^{-} /\langle\pi-1\rangle$ factors through $\mathbf{B} /\langle\pi-1\rangle$, and then we are in Kashiwara's original setting of $q$-boson algebra and its simple module. In this case, it is well known $\left([\mathrm{Ka}]\right.$ ) that crystal basis on $\mathbf{U}_{+}^{-}$exists. We shall formulate variants of the notion of crystal bases applicable to $\mathbf{U}_{\diamond}^{-}$where $\diamond \in\{\emptyset,-\}$. To that end, we consider the subcategory $\mathcal{P}_{-}$of $\mathcal{P}$ which consists of $\mathbf{B}$-modules on which $\pi$ acts as -1 , as well as the subcategory $\mathcal{P}_{\pi}$ of $\mathcal{P}$ which consists of $\mathbf{B}$-modules which are free $\mathbb{Q}(q)^{\pi}$-modules.

Let $\mathcal{A} \subseteq \mathbb{Q}(q)$ be the subring of functions regular at $q=0$. Let $R$ be an arbitrary subring of $\mathbb{Q}$ with 1 .

Definition 3.14. (1) For a free $R^{\pi}$-module $F$, a $\pi$-basis for $F$ is a subset $B$ of $F$ such that $B^{0} \subseteq B \subseteq \pi B^{0} \cup B^{0}$, for some basis $B^{0}$ of the free $R^{\pi}$-module $F$.

(2) For a free $R$-module $F$ on which $\pi$ acts as -1 , a $\pi$-basis for $F$ is a subset $B$ of $F$ such that $B^{0} \subseteq B \subseteq \pi B^{0} \cup B^{0}$, for some basis $B^{0}$ of the free $R$ module $F$. (For $F$ on which $\pi$ acts as 1 , the definition forces a $\pi$-basis to be a genuine basis.)

(3) Assume in addition that $F$ admits a nondegenerate bilinear form $(\cdot, \cdot)$. The $\pi$-basis $B$ in (1) or (2) above is called $\pi$-orthonormal if $B^{0}$ is orthogonal with respect to $(\cdot, \cdot)$ and $(b, b) \in\{1, \pi\}$ for $b \in B^{0}$.

Of course, a $\pi$-basis $B$ above gives rise to a "maximal" $\pi$-basis $\pi B^{0} \cup B^{0}$. 
Definition 3.15. A free $\mathcal{A}$-submodule $L$ of a $\mathbf{B}$-module $M$ in the category $\mathcal{P}_{-}$is called a crystal lattice if

(1) $L \otimes_{\mathcal{A}} \mathbb{Q}(q)=M$;

(2) $\tilde{e}_{i} L \subseteq L$ and $\tilde{f}_{i} L \subseteq L$.

(Note that $L / q L$ is a $\mathbb{Q}$-module.) $M$ is said to have a crystal basis $(L, B)$ if a subset $B$ of $L / q L$ satisfies

(3) $B$ is a $\pi$-basis of $L / q L$;

(4) $\tilde{e}_{i} B \subseteq B \cup\{0\}$ and $\tilde{f}_{i} B \subseteq B$;

(5) For $b \in B$, if $\tilde{e}_{i} b \neq 0$, then $b=\tilde{f}_{i} \tilde{e}_{i} b$.

A crystal basis $(L, B)$ is called maximal if $B$ is a maximal $\pi$-basis of $L / q L$.

Remark 3.16. Accordingly, a crystal $\pi$-lattice $L$ and a crystal $\pi$-basis $(L, B)$ of a $\mathbf{B}$-module $M$ in the category $\mathcal{P}_{\pi}$ consists of a free $\mathcal{A}^{\pi}$-submodule $L$ of $M$ and a subset $B$ of the $\mathbb{Q}^{\pi}$-module $L / q L$ satisfying the axioms as in Definition 3.15 with (1) modified as

(1') $L \otimes_{\mathcal{A}^{\pi}} \mathbb{Q}(q)^{\pi}=M$.

Note the meaning of $(3)$ is adjusted according to Definition 3.14

We let $\mathcal{L}(\infty)_{+}$and $\mathcal{L}(\infty)_{-}$(resp. $\left.\mathcal{L}(\infty)\right)$ be the $\mathcal{A}$-submodules of $\mathbf{U}_{+}^{-}$and $\mathbf{U}_{-}^{-}$ (respectively, the $\mathcal{A}^{\pi}$-submodule of $\mathbf{U}^{-}$) generated by all possible $\tilde{f}_{i_{1}} \ldots \tilde{f}_{i_{t}} 1$. We let $B(\infty)_{\diamond}=\left\{\tilde{f}_{i_{1}} \ldots \tilde{f}_{i_{t}} 1\right\}$ be the subset of $\mathcal{L}(\infty)_{\diamond} / q \mathcal{L}(\infty)_{\diamond}$, where $\diamond \in \operatorname{Apair}\{\emptyset,+,-\}$ and by convention again we drop the subscript $\diamond$ in case of $\diamond=\emptyset$. We shall prove in Section 5 that $\left(\mathcal{L}(\infty)_{\diamond}, B(\infty)_{\diamond}\right)$ is a crystal basis of $\mathbf{U}_{\diamond}^{-}$, for $\diamond \in\{\emptyset,+,-\}$. Note that the bilinear form allows us to define a dual lattice in $\mathbf{U}_{\diamond}^{-}$, for $\diamond \in\{\emptyset,+,-\}$ as follows:

$$
\mathcal{L}(\infty)_{ \pm}^{\vee}=\left\{u \in \mathbf{U}_{ \pm}^{-} \mid\left(u, \mathcal{L}(\infty)_{ \pm}\right) \subseteq \mathcal{A}\right\}, \quad \mathcal{L}(\infty)^{\vee}=\left\{u \in \mathbf{U}^{-} \mid(u, \mathcal{L}(\infty)) \subseteq \mathcal{A}^{\pi}\right\} .
$$

\section{Crystal bases and polarization}

In this section we formulate the main theorems of crystal bases for $\mathbf{U}^{-}$and integrable $\mathbf{U}$-modules. We also formulate the tensor product rule for crystal bases.

4.1. Kashiwara operators for U-modules. We start with the following observation.

Lemma 4.1. Let $M \in \mathcal{O}$. For each $i \in I$, every $m \in M^{\lambda}$ has a unique expression of the form

$$
m=\sum_{t \geq 0} F_{i}^{(t)} m_{t}
$$

where $m_{t} \in M^{\lambda+t \alpha_{i}} \cap \operatorname{ker} E_{i}$ are nonzero for finitely many $t$.

Proof. When $p(i)=1, M$ is a direct sum of simple $\mathbf{U}_{i}$-modules, where $\mathbf{U}_{i}$ is the quantum group of $\mathfrak{o s p}(1 \mid 2)$; see [CW, CHW]. Uniqueness is proved similarly to the case when $p(i)=0($ see $[\mathrm{Ka}, \S 2.2])$. 
Definition 4.2. Let $m \in M^{\lambda}$ with

$$
m=\sum_{t \geq 0} F_{i}^{(t)} m_{t}
$$

where $m_{t} \in M^{\lambda+t \alpha_{i}} \cap$ ker $e_{i}$ are nonzero for finitely many $t$. We define

$$
\tilde{e}_{i} m=\sum_{t} F_{i}^{(t-1)} m_{t}, \quad \tilde{f}_{i} m=\sum_{t} F_{i}^{(t+1)} m_{t} .
$$

Note that $\tilde{e}_{i} m \in M^{\lambda+\alpha_{i}}$ and $\tilde{f}_{i} m \in M^{\lambda-\alpha_{i}}$. Moreover, $\tilde{e}_{i}$ and $\tilde{f}_{i}$ (super)commute with U-module homomorphisms.

Now recall the definition of the rings $\mathcal{A}$ and $\mathcal{A}^{\pi}$ from 3.5

Definition 4.3. Let $M$ be a $\mathbf{U}$-module in the category $\mathcal{O}$. A free $\mathcal{A}$-submodule $\mathcal{L}$ of $M$ is called a crystal lattice of $M$ if

(1) $\mathcal{L} \otimes_{\mathcal{A}} \mathbb{Q}(q)=M$;

(2) $\mathcal{L}=\bigoplus_{\lambda \in P} \mathcal{L}^{\lambda}$ where $\mathcal{L}^{\lambda}=\mathcal{L} \cap M^{\lambda}$ for all $\lambda \in P$;

(3) $\tilde{e}_{i} \mathcal{L} \subseteq \mathcal{L}$ and $\tilde{f}_{i} \mathcal{L} \subseteq \mathcal{L}$.

A pair $(\mathcal{L}, B)$ is called a crystal basis of $M$ if a subset $B$ of the $\mathbb{Q}$-module $\mathcal{L} / q \mathcal{L}$ satisfies

(4) $B$ is a $\pi$-basis of $\mathcal{L} / q \mathcal{L}$ over $\mathbb{Q}$;

(5) $B=\coprod_{\lambda \in P} B^{\lambda}$ where $B^{\lambda}=B \cap \mathcal{L}^{\lambda} / q \mathcal{L}^{\lambda}$,

(6) $\tilde{e}_{i} B \subseteq B \cup\{0\}$ and $\tilde{f}_{i} B \subseteq B \cup\{0\}$;

(7) for $b, b^{\prime} \in B, \tilde{e}_{i} b=b^{\prime}$ if and only if $b=\tilde{f}_{i} b^{\prime}$.

Also, a $\pi$-version of crystal basis for $\mathbb{Q}(q)^{\pi}$-free integrable modules can be formulated similarly as in Remark 3.16 .

Remark 4.4. We shall set out to prove the existence of the crystal bases for the integrable modules $V(\lambda)_{ \pm}$, for $\lambda \in P^{+}$. Assume for the moment that we have done this. Since these axioms are unaffected under direct sums of lattices and parity changes, we can endow any integrable module $M$ with a crystal basis built out of direct sums of the simples. Uniqueness of a maximal crystal basis on $M$ (up to isomorphism) can be proved by the same arguments as in [Ka, §2.6].

Example 4.5. Let $I=\{i\}$. Then the simple modules are $(n+1)$-dimensional modules $V(n)_{ \pm}$for $n \in \mathbb{Z}_{\geq 0}$. Let $v_{n}^{+}$denote a highest weight vector in $V(n)_{ \pm}$. Define the $\mathcal{A}$-lattice $\mathcal{L}(n)_{ \pm}=\bigoplus_{k=0}^{n} \mathcal{A} F^{(k)} v_{n}^{+}$in $V(n)_{ \pm}$, and $B(n)_{ \pm}=\left\{F^{(k)} v_{n}^{+}+q \mathcal{L}(n)_{ \pm}\right.$| $0 \leq k \leq n\}$ (the index $i$ is suppressed here). Then $\left(\mathcal{L}(n)_{ \pm}, B(n)_{ \pm}\right)$is a crystal basis of $V(n)_{ \pm}$. In this case, $B(n)_{ \pm}$is actually a genuine $\mathbb{Q}$-basis for $\mathcal{L}(n)_{ \pm} / q \mathcal{L}(n)_{ \pm}$.

Example 4.6. Let $\lambda \in P^{+}$and let $v_{\lambda}^{+}$be a highest weight vector of $V(\lambda)_{ \pm}$. Consider the subset $B(\lambda)_{ \pm}:=\left\{\tilde{f}_{i_{1}} \ldots \tilde{f}_{i_{t}} v_{\lambda}^{+}\right\} \backslash\{0\}$ of $V(\lambda)_{ \pm}$. Let $\mathcal{L}(\lambda)_{ \pm}$be the $\mathcal{A}$-submodule of $V(\lambda)_{ \pm}$generated by $B(\lambda)_{ \pm}$. We shall prove in Section 5 that $\left(\mathcal{L}(\lambda)_{ \pm}, B(\lambda)_{ \pm}\right)$is a (minimal) crystal basis in contrast to the maximal crystal basis $\left(\mathcal{L}(\lambda)_{ \pm}, \pi B(\lambda)_{ \pm} \cup B(\lambda)_{ \pm}\right)$in the sense of Definition 3.14 (of course, the case of + was already in $[\mathrm{Ka}]$ ). 
Example 4.7. Assume that $I_{1}$ contains $i, j$ such that $a_{i j}=a_{j i}=0$. Then $\tilde{f}_{i} \tilde{f}_{j}=$ $\pi \tilde{f}_{j} \tilde{f}_{i}$, and hence $\pi B(\lambda)_{-} \cap B(\lambda)_{-} \neq \emptyset$ for various $\lambda \in P^{+}$.

4.2. Polarization. Let $\tau_{1}: \mathbf{U} \rightarrow \mathbf{U}$ be the anti-automorphism defined by

$$
\begin{aligned}
\tau_{1}\left(E_{i}\right) & =q_{i}^{-1} \widetilde{K}_{i}^{-1} F_{i}, \tau_{1}\left(F_{i}\right)=q_{i}^{-1} \widetilde{K}_{i} E_{i}, \\
\tau_{1}\left(K_{\mu}\right) & =K_{\mu}, \quad \tau_{1}\left(J_{\mu}\right)=J_{\mu}, \quad\left(i \in I, \mu \in P^{\vee}\right)
\end{aligned}
$$

such that $\tau_{1}(x y)=\tau_{1}(y) \tau_{1}(x)$ for $x, y \in \mathbf{U}$. One checks that $\tau_{1}^{2}=1$. (Note a typo in [Ka, §2.5] where $q_{i} t_{i}^{-1} f_{i}$ should read $q_{i}^{-1} t_{i}^{-1} f_{i}$.)

Proposition 4.8. Let $\lambda \in P^{+}$. There is a unique bilinear form $(\cdot, \cdot)$ on $V(\lambda)_{+}$ and $V(\lambda)_{-}$, respectively, which satisfies $\left(v_{\lambda}^{+}, v_{\lambda}^{+}\right)=1$ and

$$
(u v, w)=\left(v, \tau_{1}(u) w\right), \quad \forall u \in \mathbf{U}, v, w \in V(\lambda)_{ \pm}
$$

Moreover, this bilinear form on $V(\lambda)_{ \pm}$is symmetric.

Recall the $\mathcal{A}$-lattices $\mathcal{L}(\lambda)_{ \pm}$of $V(\lambda)_{ \pm}$from Example 4.6. We define the dual lattices in $V(\lambda)_{ \pm}$to be

$$
\mathcal{L}(\lambda)_{ \pm}^{\vee}=\left\{v \in V(\lambda)_{ \pm} \mid\left(v, \mathcal{L}(\lambda)_{ \pm}\right) \subseteq \mathcal{A}\right\}
$$

For a weight $\mathbf{U}$-module $M$, we call a bilinear form $(\cdot, \cdot)$ on $M$ a polarization if (4.1) is satisfied with $M$ in place of $V(\lambda)_{ \pm}$. Note that if $m \in M^{\lambda}$ and $m^{\prime} \in M^{\mu}$, then

$$
\left(m, m^{\prime}\right)=0 \text { unless } \lambda=\mu \text { and } p(m)=p\left(m^{\prime}\right) .
$$

Recall [Ka, Lemma 2.5.1] that the tensor product of modules admitting polarizations also admits a natural polarization given by the tensor of the bilinear forms. In our super setting, this is not quite true due to the additional asymmetry in the definition of the coproduct (as noted in Remark 2.3). Recall $\mathcal{O}_{ \pm}^{\text {int }}$ from $\$ 2.4$.

Lemma 4.9. Assume that either (1) $M, N \in \mathcal{O}^{\text {int }}$ are free $\mathbb{Q}(q)^{\pi}$-modules, or $(2)$ $M, N \in \mathcal{O}_{+}^{\text {int }}$, or $(3) M, N \in \mathcal{O}_{-}^{\text {int }}$. Assume $M$ and $N$ admit polarizations $(\cdot, \cdot)$. Then the symmetric bilinear form on the module $M \otimes N$ given by $\left(m_{1} \otimes n_{1}, m_{2} \otimes n_{2}\right)=$ $\left(m_{1}, m_{2}\right)\left(n_{1}, n_{2}\right)$ satisfies

$$
\left(\Delta(u)\left(m_{1} \otimes m_{2}\right), n_{1} \otimes n_{2}\right)=\left(m_{1} \otimes m_{2}, \Delta^{\prime}\left(\tau_{1}(u)\right)\left(n_{1} \otimes n_{2}\right)\right),
$$

for $u \in \mathbf{U}, m_{1}, m_{2} \in M, n_{1}, n_{2} \in N$.

We call such a bilinear form on $M \otimes N$ a $J$-polarization, as the difference on $\Delta$ and $\Delta^{\prime}$ is caused by the $J_{\mu}$ 's. 
Proof. Let $m_{1} \in M_{\mu}$ and $m_{2} \in M_{\mu^{\prime}}$. By a direct computation, we have

$$
\begin{aligned}
& \left(\Delta\left(F_{i}\right)\left(m_{1} \otimes n_{1}\right), m_{2} \otimes n_{2}\right) \\
& \quad=\left(F_{i} m_{1}, m_{2}\right)\left(n_{1}, n_{2}\right)+\pi_{i}^{p\left(m_{1}\right)} q^{\left\langle\alpha_{i}^{\vee}, \mu\right\rangle}\left(m_{1}, m_{2}\right)\left(F_{i} n_{1}, n_{2}\right)
\end{aligned}
$$

and

$$
\begin{aligned}
& \left(m_{1} \otimes n_{1}, \Delta^{\prime}\left(q_{i}^{-1} K_{i} E_{i}\right)\left(m_{2} \otimes n_{2}\right)\right) \\
& \quad=\left(m_{1}, q_{i}^{-1} K_{i} E_{i} m_{2}\right)\left(n_{1}, n_{2}\right)+\pi_{i}^{p\left(m_{2}\right)} q_{i}^{\left\langle\alpha_{i}^{\vee}, \mu^{\prime}\right\rangle}\left(m_{1}, m_{2}\right)\left(n_{1}, q_{i}^{-1} K_{i} E_{i} n_{2}\right) .
\end{aligned}
$$

By (4.2) and Proposition 4.8, (4.3) and (4.4) are equal.

Remark 4.10. To see why we need $\Delta^{\prime}$ in Lemma 4.9, we compute using $\Delta$ in place of $\Delta^{\prime}$ such that

$$
\left(m_{1} \otimes n_{1}, \Delta\left(\tau_{1}\left(E_{i}\right)\right)\left(m_{2} \otimes n_{2}\right)\right)
$$

$$
=\left(m_{1}, \tau_{1}\left(E_{i}\right) m_{2}\right)\left(n_{1}, n_{2}\right)+\pi_{i}^{p\left(m_{2}\right)}\left(\pi_{i} q_{i}\right)^{\left\langle\alpha_{i}^{\vee}, \mu^{\prime}\right\rangle}\left(m_{1}, m_{2}\right)\left(n_{1}, \tau_{1}\left(E_{i}\right) n_{2}\right) .
$$

In particular, if $p(i)=1$ and $\left\langle\alpha_{i}^{\vee}, \mu^{\prime}\right\rangle \notin 2 \mathbb{Z}$, then (4.5) is not equal to (4.3).

For $\lambda, \mu \in P^{+}$and $\diamond \in\{+,-\}$, we define the even $\mathbf{U}$-module homomorphisms

$$
\begin{aligned}
\Phi(\lambda, \mu): V(\lambda+\mu)_{\diamond} \rightarrow V(\lambda)_{\diamond} \otimes V(\mu)_{\diamond}, & v_{\lambda+\mu}^{+} \mapsto v_{\lambda}^{+} \otimes v_{\mu}^{+}, \\
\Phi^{\prime}(\lambda, \mu): V(\lambda+\mu)_{\diamond} \rightarrow V(\lambda)_{\diamond} \otimes^{\prime} V(\mu)_{\diamond}, & v_{\lambda+\mu}^{+} \mapsto v_{\lambda}^{+} \otimes v_{\mu}^{+}, \\
\Psi(\lambda, \mu): V(\lambda)_{\diamond} \otimes V(\mu)_{\diamond} \rightarrow V(\lambda+\mu)_{\diamond}, & v_{\lambda}^{+} \otimes v_{\mu}^{+} \mapsto v_{\lambda+\mu}^{+}, \\
\Psi^{\prime}(\lambda, \mu): V(\lambda)_{\diamond} \otimes^{\prime} V(\mu)_{\diamond} \rightarrow V(\lambda+\mu)_{\diamond}, & v_{\lambda}^{+} \otimes v_{\mu}^{+} \mapsto v_{\lambda+\mu}^{+} .
\end{aligned}
$$

Then $\Psi(\lambda, \mu) \circ \Phi(\lambda, \mu)$ and $\Psi^{\prime}(\lambda, \mu) \circ \Phi^{\prime}(\lambda, \mu)$ are the identity map on $V(\lambda+\mu)_{\diamond}$. In particular, $V(\lambda)_{\diamond} \otimes V(\mu)_{\diamond}=\operatorname{im} \Phi \oplus \operatorname{ker} \Psi$ and $V(\lambda)_{\diamond} \otimes^{\prime} V(\mu)_{\diamond}=\operatorname{im} \Phi^{\prime} \oplus \operatorname{ker} \Psi^{\prime}$. Note that these maps, being even $\mathbf{U}$-module homomorphisms, commute with Kashiwara operators.

Corollary 4.11. Let $\lambda, \mu \in P^{+}$and $\diamond \in\{+,-\}$. Let $(\cdot, \cdot)$ denote the polarization on $V(\lambda+\mu)_{\diamond}$ and the $J$-polarization on $V(\lambda)_{\diamond} \otimes V(\mu)_{\diamond}$. Then we have

$$
(\Psi(\lambda, \mu)(w), v)=\left(w, \Phi^{\prime}(\lambda, \mu)(v)\right), \quad\left(\Psi^{\prime}(\lambda, \mu)(w), v\right)=(w, \Phi(\lambda, \mu)(v)),
$$

for $v \in V(\lambda+\mu)_{\diamond}$ and $w \in V(\lambda)_{\diamond} \otimes V(\mu)_{\diamond}$.

4.3. Odd rank 1 calculation. Let $n \in \mathbb{Z}_{\geq 0}$ and $I=\{i\}$ with $p(i)=1$. We consider the module $V(n) \otimes V(1)$. This module has two submodules over $\mathbb{Q}(q)^{\pi}$ generated by singular vectors: a submodule $N_{1}$ generated by the (even) singular vector

$$
w=v_{n}^{+} \otimes v_{1}^{+}
$$

and $N_{2}$ generated by the (odd) singular vector

$$
z=v_{n}^{+} \otimes F v_{1}^{+}-\pi^{n} q[n]^{-1} F v_{n}^{+} \otimes v_{1}^{+} .
$$

We directly compute

$$
\begin{aligned}
F^{(k)} w & =F^{(k)} v_{n}^{+} \otimes v_{1}^{+}+\pi^{n}(\pi q)^{n+1-k} F^{(k-1)} v_{n}^{+} \otimes F v_{1}^{+}, \\
F^{(k)} z & =\left(1-(\pi q)^{n-k}[n]^{-1}[k]\right) F^{(k)} v_{n}^{+} \otimes F v_{1}^{+}-\pi^{n} q[n]^{-1}[k+1] F^{(k+1)} v_{n}^{+} \otimes v_{1}^{+} .
\end{aligned}
$$


Observing that $(\pi q)^{n-k}[n]^{-1}[k] \in q^{2 n-2 k} \mathcal{A}^{\pi}$, we have

$$
F^{(k)} w=\left\{\begin{array}{ll}
F^{(k)} v_{n}^{+} \otimes v_{1}^{+} & \text {if } 0 \leq k<n+1, \\
\pi^{n} F^{(n)} v_{n}^{+} \otimes F v_{1}^{+} & \text {if } k=n+1,
\end{array} \quad \bmod q \mathcal{L}\right.
$$

and

$$
F^{(k)} z=F^{(k)} v_{n}^{+} \otimes F v_{1}^{+} \quad \bmod q \mathcal{L}, \quad 0 \leq k \leq n-1 .
$$

In particular, $V(n) \otimes V(1) \cong N_{1} \oplus N_{2} \cong V(n+1) \oplus V(n-1)$.

The above calculations remain to make perfect sense for $V(n)_{ \pm} \otimes V(1)_{ \pm}$, with $\pi$ in the formulas above interpreted as 1 and -1 accordingly. In particular,

$$
V(n)_{ \pm} \otimes V(1)_{ \pm} \cong N_{1} \oplus N_{2} \cong V(n+1)_{ \pm} \oplus V(n-1)_{ \pm} .
$$

4.4. Tensor product rule for crystal bases. We can use the calculations in 44.3 to prove a tensor product rule for crystal bases in general. Let $M$ be an integrable U-module with crystal basis $(\mathcal{L}, B)$. For each $i \in I$ and $b \in B$, define

$$
\begin{aligned}
& \varphi_{i}(b)=\max \left\{n \mid \tilde{f}_{i}^{n} b \neq 0\right\}, \\
& \varepsilon_{i}(b)=\max \left\{n \mid \tilde{e}_{i}^{n} b \neq 0\right\} .
\end{aligned}
$$

We note that $\varphi_{i}(b)=\left\langle\alpha_{i}^{\vee}, \mu\right\rangle+\varepsilon_{i}(b)$ for $b \in B_{\mu}$.

Theorem 4.12. Let $M, M^{\prime} \in \mathcal{O}_{-}^{\text {int }}$ (resp. $M, M^{\prime} \in \mathcal{O}_{+}^{\text {int }}$ ) be modules with crystal bases $(\mathcal{L}, B)$ and $\left(\mathcal{L}^{\prime}, B^{\prime}\right)$. Let $B \otimes B^{\prime}=\left\{b \otimes b^{\prime} \in(\mathcal{L} / q \mathcal{L}) \otimes_{\mathbb{Q}}\left(\mathcal{L}^{\prime} / q \mathcal{L}^{\prime}\right): b \in B, b^{\prime} \in B^{\prime}\right\}$. The tensor product $M \otimes M^{\prime}$ has a crystal basis $\left(\mathcal{L} \otimes \mathcal{L}^{\prime}, B \otimes B^{\prime}\right)$ subject to the rules:

$$
\begin{aligned}
& \tilde{f}_{i}\left(b \otimes b^{\prime}\right)= \begin{cases}\tilde{f}_{i} b \otimes b^{\prime} & \text { if } \varphi_{i}(b)>\varepsilon_{i}\left(b^{\prime}\right), \\
\pi_{i}^{p(b)} b \otimes \tilde{f}_{i} b^{\prime} & \text { otherwise } ;\end{cases} \\
& \tilde{e}_{i}\left(b \otimes b^{\prime}\right)= \begin{cases}\pi_{i}^{p(b)} b \otimes \tilde{e}_{i} b^{\prime} & \text { if } \varphi_{i}(b)<\varepsilon_{i}\left(b^{\prime}\right), \\
\tilde{e}_{i} b \otimes b^{\prime} & \text { otherwise. }\end{cases}
\end{aligned}
$$

(All equalities are understood in $\mathcal{L} \otimes \mathcal{L}^{\prime} / q \mathcal{L} \otimes \mathcal{L}^{\prime}$.)

Proof. It is sufficient to prove this for a fixed $i$, in which case the theorem reduces to a statement for $I=\{i\}$. When $p(i)=0$, the theorem is [Ka, Theorem 1]. Assume $p(i)=1$. The case for $M, M^{\prime} \in \mathcal{O}_{+}^{\text {int }}$ is again reduced to Kashiwara's original setting, so we assume $M, M^{\prime} \in \mathcal{O}_{-}^{\text {int }}$. Since modules are completely reducible [CW], it suffices to prove this for tensor products of simple modules $V(n)_{-} \otimes V(m)_{-}$, by induction on $m$. Recall from Example 4.5 that $\left(\mathcal{L}(n)_{ \pm}, B(n)_{ \pm}\right)$is a crystal basis for $V(n)_{ \pm}$.

From the odd rank 1 calculation in $\$ 4.3$, the theorem holds for $V(n)_{-} \otimes V(1)_{-}$. This takes care of the base case of induction.

By induction, we assume the theorem holds for $V(n)_{-} \otimes V(m)_{-}$. Note that

$$
V(n)_{-} \otimes V(m)_{-} \otimes V(1)_{-} \cong V(n)_{-} \otimes\left(V(m+1)_{-} \oplus V(m-1)_{-}\right) .
$$

By the complete reducibility and the base case proved above, we conclude that $\left(\mathcal{L}(n)_{-} \otimes \mathcal{L}(m)_{-} \otimes \mathcal{L}(1)_{-}, B(n)_{-} \otimes B(m)_{-} \otimes B(1)_{-}\right)$is a crystal basis of $V(n)_{-} \otimes$ $V(m)_{-} \otimes V(1)_{-}$. Moreover, $\left(\mathcal{L}(n)_{-} \otimes \mathcal{L}(m)_{-} \otimes \mathcal{L}(1)_{-}, B(n)_{-} \otimes B(m)_{-} \otimes B(1)_{-}\right)$ 
decomposes as $\left(\mathcal{L}(n)_{-} \otimes\left(\mathcal{L}(m+1)_{-} \oplus \mathcal{L}(m-1)_{-}\right), B(n)_{-} \otimes\left(B(m+1)_{-} \cup\right.\right.$ $\left.\left.B(m-1)_{-}\right)\right)$. Therefore, $\left(\mathcal{L}(n)_{-} \otimes \mathcal{L}(m+1)_{-}, B(n)_{-} \otimes B(m+1)_{-}\right)$is a crystal basis of $V(n)_{-} \otimes V(m+1)_{-}$.

Remark 4.13. Jeong Jeo claimed a version of tensor product rule for $\mathfrak{o s p}(1 \mid 2)$ without super signs. The proof was much more complicated in the setting of [Jeo], as $V(n)$ for $n$ odd (and in particular $V(1)$ ) were not available. The formulation and the calculations there were incorrect since he missed the super signs in the quantum integers and the super signs arising from the multiplication in the tensor algebra; see (2.11). For example, the correct calculation of [Jeo, Eq. (4.2)] should read (it is understood that $\pi=-1$ below)

$$
a_{m}=\frac{1}{[m]} \sum_{k=0}^{m-1}\left(\pi q^{-2}\right)^{k} q^{n}=\pi^{m-1} q^{n+1-m} .
$$

In particular, $\tilde{f}^{n+1}\left(v_{n}^{+} \otimes v_{2}^{+}\right) \equiv \pi^{n} \tilde{f}^{n} v_{n}^{+} \otimes \tilde{f}_{i} v_{1}^{+} \bmod q A$.

On the other hand, there are no signs in the tensor product rule presented in [MZ, Theorem 4.1], since the signs are absorbed into the various factors of $\sqrt{-1}$ therein.

4.5. Main results on crystal bases. We are now ready to formulate the main theorems on crystal bases for $\mathbf{U}^{-}$and integrable modules $V(\lambda)$.

Theorem 4.14. For $\diamond \in\{\emptyset,+,-\},\left(\mathcal{L}(\infty)_{\diamond}, B(\infty)_{\diamond}\right)$ is a crystal basis of $\mathbf{U}_{\diamond}^{-}$.

Theorem 4.15. Let $\lambda \in P^{+}$. For $\diamond \in\{+,-, \emptyset\},\left(\mathcal{L}(\lambda)_{\diamond}, B(\lambda)_{\diamond}\right)$ is a crystal basis of $V(\lambda)_{\diamond}$.

For $\lambda \in P^{+}$and $\diamond \in\{\emptyset,+,-\}$, we define the (even) $\mathbf{U}^{-}$-linear projection map

$$
\wp_{\lambda}: \mathbf{U}_{\diamond}^{-} \longrightarrow V(\lambda)_{\diamond}, \quad 1 \mapsto v_{\lambda}^{+} \text {. }
$$

Theorem 4.16. Let $\lambda \in P^{+}$. For $\diamond \in\{+,-, \emptyset\}$,

(1) $\wp_{\lambda}\left(\mathcal{L}(\infty)_{\diamond}\right)=\mathcal{L}(\lambda)_{\diamond} ;$

(This induces a homomorphism $\hat{\wp}_{\lambda}: \mathcal{L}(\infty)_{\diamond} / q \mathcal{L}(\infty)_{\diamond} \rightarrow \mathcal{L}(\lambda)_{\diamond} / q \mathcal{L}(\lambda)_{\diamond}$.)

(2) $\hat{\wp}_{\lambda}$ sends $\left\{b \in B(\infty)_{\diamond} \mid \hat{\wp}_{\lambda}(b) \neq 0\right\}$ isomorphically to $B(\lambda)_{\diamond}$;

(3) if $b \in B(\infty)_{\diamond}$ satisfies $\hat{\wp}_{\lambda}(b) \neq 0$, then $\tilde{e}_{i} \hat{\wp}_{\lambda}(b)=\hat{\wp}_{\lambda}\left(\tilde{e}_{i} b\right)$.

The proofs of these three theorems on crystal bases will be given in the following section.

\section{THE GRAND LOOP ARGUMENT}

In this section, $\pi$ is understood as its specialization to -1 , and we adopt the convention (in this section only) of dropping the subscript "-" everywhere in $V(\lambda), \mathcal{L}(\lambda), B(\lambda), \mathbf{U}, \mathbf{U}^{-}, \mathcal{L}(\infty), B(\infty)$ for all $\lambda$ for notational simplicity. (If instead we specialize $\pi$ to 1 and add the subscript "+" everywhere, we would be back to the setting of Kashiwara [Ka, §4]). We prove the existence of crystal bases using a modified version of Kashiwara's grand loop argument, and will present only the parts which differ most from [Ka, §4]. 
For $\lambda, \mu \in P^{+}$, recall the maps $\Psi(\lambda, \mu)$ and $\Phi(\lambda, \mu)$ from (4.6). Note that these maps, being (even) U-module homomorphisms, commute with the Kashiwara operators. We also define a map

$$
S(\lambda, \mu): V(\lambda) \otimes V(\mu) \longrightarrow V(\lambda)
$$

by $S(\lambda, \mu)\left(u \otimes v_{\mu}^{+}\right)=u$ and $S(\lambda, \mu)\left(V(\lambda) \otimes \sum f_{i} V(\mu)\right)=0$. This is an (even) $\mathbf{U}^{-}$-linear map. Therefore, we have an (even) $\mathbf{U}^{-}$-linear map $S(\lambda, \mu) \circ \Phi(\lambda, \mu)$ : $V(\lambda+\mu) \longrightarrow V(\lambda)$ sending $v_{\lambda+\mu}^{+}$to $v_{\lambda}^{+}$.

For $\zeta \in Q^{-}$, set

$$
\text { ht } \zeta=\sum n_{i} \text { if } \zeta=-\sum n_{i} \alpha_{i} .
$$

Then let $Q^{-}(l)=\left\{\zeta \in Q^{-}\right.$: ht $\left.\zeta \leq l\right\}$.

Let $C_{l}$ be the collection of the following statements.

$\left(C_{l} .1\right)$ For $\zeta \in Q^{-}(l)$,

$$
\tilde{e}_{i} \mathcal{L}(\infty)^{\zeta} \subseteq \mathcal{L}(\infty)
$$

$\left(C_{l} .2\right)$ For $\zeta \in Q^{-}(l)$ and $\lambda \in P^{+}$,

$$
\tilde{e}_{i} \mathcal{L}(\lambda)^{\lambda+\zeta} \subset \mathcal{L}(\lambda) .
$$

$\left(C_{l} .3\right)$ For $\zeta \in Q^{-}(l)$ and $\lambda \in P^{+}$,

$$
\wp_{\lambda} \mathcal{L}(\infty)^{\zeta}=\mathcal{L}(\lambda)^{\lambda+\zeta} .
$$

$\left(C_{l} .4\right)$ For $\zeta \in Q^{-}(l), B(\infty)^{\zeta}$ is a $\pi$-basis of $\mathcal{L}(\infty)^{\zeta} / q \mathcal{L}(\infty)^{\zeta}$.

$\left(C_{l} .5\right)$ For $\zeta \in Q^{-}(l)$ and $\lambda \in P^{+}, B(\lambda)^{\lambda+\zeta}$ is a $\pi$-basis of $\mathcal{L}(\lambda)^{\lambda+\zeta} / q \mathcal{L}(\lambda)^{\lambda+\zeta}$.

$\left(C_{l} .6\right)$ For $\zeta \in Q^{-}(l-1)$ and $\lambda \in P^{+}, \tilde{f}_{i}\left(x v_{\lambda}^{+}\right) \equiv\left(\tilde{f}_{i} x\right) v_{\lambda}^{+} \bmod q \mathcal{L}(\lambda)$ for $x \in$ $\mathcal{L}(\infty)^{\zeta}$.

$\left(C_{l} .7\right)$ For $\zeta \in Q^{-}(l)$ and $\lambda \in P^{+}, \tilde{e}_{i} B(\infty)^{\zeta} \subset B(\infty) \cup\{0\}$ and $\tilde{e}_{i} B(\lambda)^{\lambda+\zeta} \subset$ $B(\lambda) \cup\{0\}$.

$\left(C_{l} .8\right)$ For $\zeta \in Q^{-}(l)$ and $\lambda, \mu \in P^{+}$,

$$
\Phi(\lambda, \mu)\left(\mathcal{L}(\lambda+\mu)^{\lambda+\mu+\zeta}\right) \subset \mathcal{L}(\lambda) \otimes \mathcal{L}(\mu) .
$$

$\left(C_{l} .9\right)$ For $\zeta \in Q^{-}(l)$ and $\lambda, \mu \in P^{+}$,

$$
\Psi(\lambda, \mu)\left((\mathcal{L}(\lambda) \otimes \mathcal{L}(\mu))^{\lambda+\mu+\zeta}\right) \subset \mathcal{L}(\lambda+\mu) .
$$

$\left(C_{l} .10\right)$ For $\zeta \in Q^{-}(l)$ and $\lambda, \mu \in P^{+}$,

$$
\Psi(\lambda, \mu)\left((B(\lambda) \otimes B(\mu))^{\lambda+\mu+\zeta}\right) \subset B(\lambda+\mu) \cup\{0\} .
$$

$\left(C_{l} .11\right)$ For $\zeta \in Q^{-}(l)$ and $\lambda \in P^{+}$,

$$
\left\{b \in B(\infty)^{\zeta}: \hat{\wp}_{\lambda}(b) \neq 0\right\} \rightarrow B(\lambda)^{\lambda+\zeta}
$$

is a bijection, where $\hat{\wp}_{\lambda}$ is the map induced by $\wp_{\lambda}$.

$\left(C_{l} .12\right)$ For $\zeta \in Q^{-}(l), \lambda \in P^{+}$and $b \in B(\infty)^{\zeta}$ such that $\hat{\wp}_{\lambda}(b) \neq 0$, we have

$$
\tilde{e}_{i} \hat{\wp}_{\lambda}(b)=\hat{\wp}_{\lambda}\left(\tilde{e}_{i}(b)\right) \text {. }
$$

$\left(C_{l} .13\right)$ For $\zeta \in Q^{-}(l), \lambda \in P^{+} b \in B(\lambda)^{\lambda+\zeta}$ and $b^{\prime} \in B(\lambda)^{\lambda+\zeta+\alpha_{i}}, b=\tilde{f}_{i} b^{\prime}$ if and only if $b^{\prime}=\tilde{e}_{i} b$.

$\left(C_{l} .14\right)$ For $\zeta \in Q^{-}(l)$ and $b \in B(\infty)$, if $\tilde{e}_{i} b \neq 0$, then $b=\tilde{f}_{i} \tilde{e}_{i} b$. 
In the case $I=I_{0}$, Kashiwara (cf. [Ka] proved these statements via an induction on $l$. These arguments can be adapted to our super setting, with the main change being bookkeeping for the power of $\pi$; we will formulate precisely the $\pi$-modified results with proofs. It is worth noting that Lemmas 5.55 5.7 (corresponding to $\mathrm{Ka}$, Lemmas 4.7.1-4.7.3]) deviate the most in the general case. This is a consequence of the failure of the natural bilinear form on tensors to necessarily be a polarization; see Lemma 4.9

Lemma 5.1 ([ $\overline{\mathrm{Ka}}$, Lemma 4.3.1]). Let $\zeta \in Q^{-}(l-1), \lambda \in P^{+}$and $u \in \mathcal{L}(\infty)^{\zeta}$ (resp. $u \in \mathcal{L}(\lambda)^{\lambda+\zeta}$ ). If $u=\sum F_{i}^{(n)} u_{n}$ and if $e_{i} u_{n}=0$ (resp. $u_{n} \in V(\lambda)^{\lambda+\zeta+n \alpha_{i}}$, $E_{i} u_{n}=0$ and $u_{n}=0$ except when $\left.\left\langle\alpha_{i}^{\vee}, \lambda+\zeta+n \alpha_{i}\right\rangle \geq n\right)$, then all $u_{n}$ belong to $\mathcal{L}(\infty)$ (resp. $\mathcal{L}(\lambda))$.

If, moreover, $u \bmod q \mathcal{L}(\infty)$ (resp. $q \mathcal{L}(\lambda)$ belongs to $B(\infty)$ (resp. $B(\lambda)$ ), then there exists $n$ such that $u=f_{i}^{(n)} u_{n} \bmod q \mathcal{L}(\infty)($ resp. $q \mathcal{L}(\lambda))$.

Proof. By $\left(C_{l-1} .1\right), \tilde{e}_{i}^{t} u \in \mathcal{L}(\infty)$ for all $t$. Let $m$ be the largest integer such that $u_{m} \notin \mathcal{L}(\infty)$. Then $\tilde{e}_{i}^{m} u=\sum_{n \geq 0} f_{i}^{(n-m)} u_{n}$. Since $u_{n} \in \mathcal{L}(\infty)$ for $n>m, u_{m} \in$ $\mathcal{L}(\infty)$, a contradiction. Therefore, $u_{n} \in \mathcal{L}(\infty)$ for all $n$. A similar proof applies to $\mathcal{L}(\lambda)$.

Now suppose $u+q \mathcal{L}(\infty) \in B(\infty)$. Let $n$ be the largest integer such that $u_{n} \notin$ $q \mathcal{L}(\infty)$. Then $\tilde{e}_{i}^{n} u+q \mathcal{L}(\infty)=u_{n}+q \mathcal{L}(\infty) \neq q \mathcal{L}(\infty)$. By $\left(C_{k} .14\right)$ for $k \leq l-1$, $u+q \mathcal{L}(\infty)=\tilde{f}_{i}^{n} \tilde{e}_{i}^{n} u+q \mathcal{L}(\infty)=f_{i}^{(n)} u_{n}+q \mathcal{L}(\infty)$.

Recall the map $\varepsilon_{i}$ from (4.9). By the previous lemma, for $\zeta \in Q^{-}(l-1)$ and $b \in$ $B(\infty)^{\zeta}$ (resp. $\left.b \in B(\lambda)^{\zeta+\lambda}\right)$, there exists $u \in \mathcal{L}(\infty)^{\zeta+n \alpha_{i}}$ (resp. $u \in \mathcal{L}(\lambda)^{\lambda+\zeta+n \alpha_{i}}$ ) such that $e_{i} u=0$ and $b+q \mathcal{L}(\infty)=f_{i}^{\left(\varepsilon_{i}(b)\right)} u+q \mathcal{L}(\infty)\left(\right.$ resp. $b+q \mathcal{L}(\lambda)=f_{i}^{\left(\varepsilon_{i}(b)\right)} u+$ $q \mathcal{L}(\lambda))$; moreover, $u+q \mathcal{L}(\infty) \in B(\infty)$ (resp. $u+q \mathcal{L}(\lambda) \in B(\lambda)$ ).

The following is a $\pi$-analogue of [Ka, Lemma 4.3.2].

Lemma 5.2. Let $\zeta, \zeta^{\prime} \in Q^{-}(l-1), \lambda, \mu \in P^{+}$and $i \in I$.

(i) $\tilde{f}_{i}\left(\mathcal{L}(\lambda)^{\lambda+\zeta} \otimes \mathcal{L}(\mu)^{\mu+\zeta^{\prime}}\right) \subset \mathcal{L}(\lambda) \otimes \mathcal{L}(\mu)$ and $\tilde{e}_{i}\left(\mathcal{L}(\lambda)^{\lambda+\zeta} \otimes \mathcal{L}(\mu)^{\mu+\zeta^{\prime}}\right) \subset$ $\mathcal{L}(\lambda) \otimes \mathcal{L}(\mu)$.

(ii) If $b \in B(\lambda)^{\lambda+\zeta}$ and $b^{\prime} \in B(\mu)^{\mu+\zeta^{\prime}}$, then we have

$$
\begin{aligned}
& \tilde{f}_{i}\left(b \otimes b^{\prime}\right)= \begin{cases}\tilde{f}_{i} b \otimes b^{\prime} & \text { if }\left\langle\alpha_{i}^{\vee}, \lambda+\zeta\right\rangle+\varepsilon_{i}(b)>\varepsilon_{i}\left(b^{\prime}\right), \\
\pi_{i}^{p(b)} b \otimes \tilde{f}_{i} b^{\prime} & \text { otherwise; }\end{cases} \\
& \tilde{e}_{i}\left(b \otimes b^{\prime}\right)= \begin{cases}\pi_{i}^{p(b)} b \otimes \tilde{e}_{i} b^{\prime} & \text { if }\left\langle\alpha_{i}^{\vee}, \lambda+\zeta\right\rangle+\varepsilon_{i}(b)<\varepsilon_{i}\left(b^{\prime}\right), \\
\tilde{e}_{i} b \otimes b^{\prime} & \text { otherwise, }\end{cases}
\end{aligned}
$$

where all equalities are in $\mathcal{L}(\lambda) \otimes \mathcal{L}(\mu) / q \mathcal{L}(\lambda) \otimes \mathcal{L}(\mu)$.

(iii) For $b \otimes b^{\prime} \in B(\lambda)^{\lambda+\zeta} \otimes B(\mu)^{\mu+\zeta^{\prime}}, \tilde{e}_{i}\left(b \otimes b^{\prime}\right) \neq 0$ implies that $b \otimes b^{\prime}=$ $\tilde{f}_{i} \tilde{e}_{i}\left(b \otimes b^{\prime}\right)$.

(iv) For $b \in B(\lambda)^{\lambda+\zeta}$ and $b^{\prime} \in B(\mu)^{\mu+\zeta^{\prime}}$, if $\tilde{e}_{i}\left(b \otimes b^{\prime}\right)=0$ for any $i$, then $\zeta=0$ and $b=v_{\lambda}^{+}+q \mathcal{L}(\lambda)$ or $b=\pi v_{\lambda}^{+}+q \mathcal{L}(\lambda)$.

(v) For $b \in B(\lambda)^{\lambda+\zeta}, \tilde{f}_{i}\left(b \otimes v_{\mu}^{+}\right)=\tilde{f}_{i} b \otimes v_{\mu}^{+}$or $\tilde{f}_{i} b=0$. 
Proof. (i). By Lemma [5.1] it is enough to show that for $u \in \mathcal{L}(\lambda)^{\lambda+\zeta+n i^{\prime}}$ and $u^{\prime} \in \mathcal{L}(\mu)^{\mu+\zeta^{\prime}+m \alpha_{i}}$ such that $E_{i} u=0, E_{i} u^{\prime}=0,\left\langle\alpha_{i}^{\vee}, \lambda+\zeta+n \alpha_{i}\right\rangle \geq n$ and $\left\langle\alpha_{i}^{\vee}, \lambda+\zeta+m \alpha_{i}\right\rangle \geq m$, then

$$
\begin{aligned}
& \tilde{f}_{i}\left(F_{i}^{(n)} u \otimes F_{i}^{(m)} u^{\prime}\right) \in \mathcal{L}(\lambda) \otimes \mathcal{L}(\mu), \\
& \tilde{e}_{i}\left(F_{i}^{(n)} u \otimes F_{i}^{(m)} u^{\prime}\right) \in \mathcal{L}(\lambda) \otimes \mathcal{L}(\mu) .
\end{aligned}
$$

This is a rank 1 calculation, and follows from the tensor product rule (Theorem 4.12).

(ii)-(v). These follow immediately from Part (i), Lemma [5.1, and Theorem 4.12 .

Corollary 5.3 (cf. [Ka, Corollary 4.3.5]). For $i_{1}, \ldots, i_{l} \in I$ and $\mu \in P^{+}$, let $\lambda=\omega_{l-1}$. Then

$$
\tilde{f}_{i_{1}} \ldots \tilde{f}_{i_{l}}\left(v_{\lambda}^{+} \otimes v_{\mu}^{+}\right)+q \mathcal{L}(\lambda) \otimes \mathcal{L}(\mu)=v \otimes w+q \mathcal{L}(\lambda) \otimes \mathcal{L}(\mu),
$$

where $v \in B(\lambda)^{\lambda+\zeta}$ and $w \in B(\mu)^{\mu+\zeta^{\prime}} \cup\{0\}$ for some $\zeta, \zeta^{\prime} \in Q^{-}(l-1) \backslash\{0\}$.

Proof. Assume that $i_{l} \neq i_{l-1}$. Then $\tilde{f}_{i_{l}} v_{\lambda}^{+}=0$, so

$$
\tilde{f}_{i_{l}}\left(v_{\lambda}^{+} \otimes v_{\mu}^{+}\right)=f_{i_{l}}\left(v_{\lambda}^{+} \otimes v_{\mu}^{+}\right)=q_{i_{l}}^{\left\langle\alpha_{i_{l}}, \omega_{i_{l-1}}\right\rangle} v_{\lambda}^{+} \otimes f_{i_{l}} v_{\mu}^{+}=v_{\lambda}^{+} \otimes \tilde{f}_{i_{l}} v_{\mu}^{+} .
$$

Then $\tilde{e}_{i_{l-1}} \tilde{f}_{i_{l}} v_{\mu}^{+}=0$ and $\tilde{f}_{i_{l-1}} v_{\lambda}^{+}=f_{i_{l-1}} v_{\lambda}^{+} \neq 0$ whence

$$
\tilde{f}_{i_{l-1}} \tilde{f}_{i_{l}}\left(v_{\lambda}^{+} \otimes v_{\mu}^{+}\right)=\left(\tilde{f}_{i_{l-1}} v_{\lambda}^{+}\right) \otimes\left(\tilde{f}_{i_{l}} v_{\mu}^{+}\right) \bmod q \mathcal{L}(\lambda) \otimes \mathcal{L}(\mu) .
$$

If $i_{l-1}=i_{l}$, then since $\tilde{f}_{i_{l}}^{2} v_{\lambda}^{+}=0$,

$$
\tilde{f}_{i_{l}}^{2}\left(v_{\lambda}^{+} \otimes v_{\mu}^{+}\right)=\pi_{i_{l}}^{p\left(i_{l}\right)}\left(\tilde{f}_{i_{l}} v_{\lambda}^{+}\right) \otimes\left(\tilde{f}_{i_{l}} v_{\mu}^{+}\right) \bmod q \mathcal{L}(\lambda) \otimes \mathcal{L}(\mu) .
$$

Then by Lemma 5.2 (ii), the assertion follows.

Corollary 5.4 (cf. Ka, Corollary 4.3.6]). Let $\lambda, \mu \in P^{+}$and $\zeta \in Q^{-}(l)$. Then

$$
(\mathcal{L}(\lambda) \otimes \mathcal{L}(\mu))^{\lambda+\mu+\zeta}=\sum_{i} \tilde{f}_{i}(\mathcal{L}(\lambda) \otimes \mathcal{L}(\mu))^{\lambda+\mu+\zeta+\alpha_{i}}+v_{\lambda}^{+} \otimes \mathcal{L}(\mu)^{\mu+\zeta} .
$$

Proof. Let $\mathcal{L}$ denote the left-hand side and $\tilde{\mathcal{L}}$ denote the right-hand side of the above desired identity. It is clear that $\tilde{\mathcal{L}} \subseteq \mathcal{L}$.

For $\zeta^{\prime} \in Y^{-}(l-1) \backslash\{0\}$ and $b \in B(\lambda)^{\lambda+\zeta^{\prime}} \otimes B(\mu)^{\mu+\zeta-\zeta^{\prime}}$, then there exists $i \in I$ with $\tilde{e}_{i} b \neq 0$ by Lemma 5.2 (iv), whence $b=\tilde{f}_{i} \tilde{e}_{i} b$ by part (iii). Therefore, $\mathcal{L}(\lambda)^{\lambda+\zeta^{\prime}} \otimes \mathcal{L}(\mu)^{\mu+\zeta-\zeta^{\prime}} \subset \tilde{\mathcal{L}}+q \mathcal{L}$ and thus

$$
\mathcal{L} \subset \tilde{\mathcal{L}}+\mathcal{L}(\lambda) \otimes v_{\mu}^{+}+q \mathcal{L} .
$$

Now, for $\tilde{f}_{i_{1}} \ldots \tilde{f}_{i_{l}} v_{\lambda}^{+} \in B(\lambda)^{\lambda+\zeta}$, we have

$$
\left(\tilde{f}_{i_{1}} \ldots \tilde{f}_{i_{l}} v_{\lambda}^{+}\right) \otimes v_{\mu}^{+}=\tilde{f}_{i_{1}}\left(\left(\tilde{f}_{i_{2}} \ldots \tilde{f}_{i_{l}} v_{\lambda}^{+}\right) \otimes v_{\mu}^{+}\right) \bmod q \mathcal{L}(\lambda) \otimes \mathcal{L}(\mu)
$$

and hence $\mathcal{L} \subset \tilde{\mathcal{L}}+q \mathcal{L}$. But $q \in \operatorname{Rad}\left(\mathcal{A}^{\pi}\right)$, the Jacobson radical of $\mathcal{A}^{\pi}$, hence by Nakayama's lemma, $\mathcal{L}=\tilde{\mathcal{L}}$. 
Lemma 5.5 (compare [Ka, Lemma 4.7.1]). For $\zeta=-\sum n_{i} \alpha_{i} \in Q^{-}$and $P, Q \in$ $\mathbf{U}^{-}$, there exists a polynomial $f\left(x_{1}, \ldots, x_{n}\right)$ in $x=\left(x_{i}\right)_{i \in I}$ with coefficients in $\mathbb{Q}(q)^{\pi}$ such that

$$
\begin{gathered}
\left(P v_{\lambda}^{+}, Q v_{\lambda}^{+}\right)=f(x) \quad \text { with } x_{i}=\left(\pi_{i} q_{i}^{2}\right)^{\left\langle\alpha_{i}^{\vee}, \lambda\right\rangle}, \text { and } \\
f(0)=\left(\prod\left(1-\pi_{i} q_{i}^{2}\right)^{-n_{i}}\right)(P, Q) .
\end{gathered}
$$

For a weight $\lambda \in P^{+}$, we write that $\lambda \gg 0$ if $\lambda-\zeta \geq 0$ for all $\zeta \in Q^{-}(l)$.

Lemma 5.6 ([Ka, Lemma 4.7.2]). For $\mu \gg 0, \wp_{\mu}\left(\mathcal{L}(\infty)^{\zeta, \vee}\right)=\mathcal{L}(\mu)^{\mu+\zeta, \vee}$ for $\zeta \in Q^{-}(l)$.

Recall the comultiplication $\Delta^{\prime}$ from (2.14) was used in defining the operators $\Phi^{\prime}$ and $\Psi^{\prime}$, just as $\Delta$ was used in defining the operators $\Phi$ and $\Psi$; see (4.6) and Lemma 4.9.

Lemma 5.7 (compare [Ka, Proposition 4.7.3]). Let $\lambda, \mu \in P^{+}$with $\mu \gg 0$ and let $\zeta \in Q^{-}(l)$. Then

$$
\Psi(\lambda, \mu)\left((\mathcal{L}(\lambda) \otimes \mathcal{L}(\mu))^{\lambda+\mu+\zeta}\right)=\mathcal{L}(\lambda+\mu)^{\lambda+\mu+\zeta} .
$$

Proof. In this proof, we shall need $\Phi^{\prime}(\lambda, \mu)$ instead of $\Phi(\lambda, \mu)$ as in [Ka].

We have $\wp_{\lambda+\mu}\left(\mathcal{L}(\infty)^{\zeta}\right)=\mathcal{L}(\lambda+\mu)^{\lambda+\mu+\zeta}$ and $\wp_{\mu}\left(\mathcal{L}(\infty)^{\zeta, \vee}\right)=\mathcal{L}(\mu)^{\mu+\zeta, \vee}$. We have

$$
(\mathcal{L}(\lambda) \otimes \mathcal{L}(\mu))^{\lambda+\mu+\zeta}=\sum \tilde{f}_{i}(\mathcal{L}(\lambda) \otimes \mathcal{L}(\mu))^{\lambda+\mu+\zeta+\alpha_{i}}+v_{\lambda}^{+} \otimes \mathcal{L}(\mu)^{\mu+\zeta} .
$$

Now for $u \in \mathcal{L}(\lambda+\mu)^{\lambda+\mu+\zeta, \vee}$, we have

$$
\begin{aligned}
\left(\Phi^{\prime}(\lambda, \mu)\right. & \left.(u), \tilde{f}_{i}(\mathcal{L}(\lambda) \otimes \mathcal{L}(\mu))^{\lambda+\mu+\zeta+\alpha_{i}}\right) \\
= & \left(u, \tilde{f}_{i} \Psi(\lambda, \mu)(\mathcal{L}(\lambda) \otimes \mathcal{L}(\mu))^{\lambda+\mu+\zeta+\alpha_{i}}\right) \\
& \subset\left(u, \tilde{f}_{i} \mathcal{L}(\lambda+\mu)^{\lambda+\mu+\zeta+\alpha_{i}}\right) \subset \mathcal{A} .
\end{aligned}
$$

Let $u=P v_{\lambda+\mu}^{+}$with $P \in \mathcal{L}(\infty)^{\zeta, \vee}$. Then for $\zeta=-\sum n_{i} \alpha_{i}$, we have

$$
\Delta^{\prime}(P)=\left(\prod\left(\widetilde{J}_{i} \widetilde{K}_{i}\right)^{n_{i}}\right) \otimes P \bmod \left(\sum F_{i} \mathbf{U}^{\leq 0}\right) \otimes \mathbf{U}^{-} .
$$

Therefore we have

$$
\Phi^{\prime}(\lambda, \mu)\left(P v_{\lambda+\mu}^{+}\right)=\left(\prod\left(\pi_{i} q_{i}\right)^{n_{i}\left\langle\alpha_{i}^{\vee}, \lambda\right\rangle}\right) v_{\lambda}^{+} \otimes P v_{\mu}^{+} \bmod \left(\sum F_{i} V(\lambda)\right) \otimes V(\mu)
$$

and thus

$$
\left(\Phi^{\prime}(\lambda, \mu)(u), v_{\lambda}^{+} \otimes \mathcal{L}(\mu)\right) \subseteq\left(\prod\left(\pi_{i} q_{i}\right)^{n_{i}\left\langle\alpha_{i}^{\vee}, \lambda\right\rangle}\right)\left(P v_{\mu}^{+}, \mathcal{L}(\mu)^{\mu+\zeta}\right) \subset \mathcal{A} .
$$

So we have shown

$$
\left(\mathcal{L}(\lambda+\mu)^{\lambda+\mu+\zeta, \vee}, \Psi(\lambda, \mu)(\mathcal{L}(\lambda) \otimes \mathcal{L}(\mu))^{\lambda+\mu+\zeta}\right) \subset \mathcal{A}
$$

and thus $\Psi(\lambda, \mu)\left(\mathcal{L}(\lambda) \otimes \mathcal{L}(\mu)^{\lambda+\mu+\zeta}\right) \subset \mathcal{L}(\lambda+\mu)^{\lambda+\mu+\zeta}$. Since $\Psi(\lambda, \mu) \circ \Phi(\lambda, \mu)$ is the identity, the reverse inclusion also holds. 
The other parts of Kashiwara's grand loop argument works equally well in our current setting. Summarizing, Kashiwara's grand loop argument in [Ka, §4] with the above modifications goes through, and we have established $\left(C_{l} .1\right)-\left(C_{l} .14\right)$.

Clearly the validity of $\left(C_{l} .1\right)-\left(C_{l} .14\right)$ implies Theorems 4.144 .16 in 84.5 for $\diamond=-$. Also, these three theorems in $\$ 4.5$ hold for $\diamond=+$ by $\mathrm{Ka}$. The crystal bases involved in Theorems 4.14 4.16 are formally defined by the same formulas. Hence we can lift the crystal bases in the cases $\diamond= \pm$ to a formal parameter $\pi$ with $\pi^{2}=1$, and we conclude that Theorems 4.14,4.16 hold in the case $\diamond=\emptyset$ as well.

\section{Properties of polarization}

Let us examine more closely the properties of the polarizations on $\mathcal{L}(\lambda)$ and on $\mathcal{L}(\infty)$

6.1. The $\pi$-orthonormality of polarizations. Recall the notion of ( $\pi$-orthonormal) $\pi$-basis from Definition 3.14

Proposition 6.1. For $\diamond \in\{+,-\}$, let either $(1) V=\mathbf{U}_{\diamond}^{-}$and $(\mathcal{L}, B)=\left(\mathcal{L}(\infty)_{\diamond}\right.$, $\left.B(\infty)_{\diamond}\right)$ or $(2) V=V(\lambda)_{\diamond}$ and $(\mathcal{L}, B)=\left(\mathcal{L}(\lambda)_{\diamond}, B(\lambda)_{\diamond}\right)$ for $\lambda \in P^{+}$. Let $(\cdot, \cdot)$ be the polarization on $V$ given in $\$ 3.4$ or $\$ 4.2$, accordingly. Then,

(i) $(\mathcal{L}, \mathcal{L}) \subseteq \mathcal{A}$, and so it descends to a bilinear form

$$
(\cdot, \cdot)_{0}: \mathcal{L} / q \mathcal{L} \times \mathcal{L} / q \mathcal{L} \rightarrow \mathbb{Q}, \quad(x+q \mathcal{L}, y+q \mathcal{L})_{0}=\left.(x, y)\right|_{q=0} .
$$

(ii) $\left(\tilde{f}_{i} u, v\right)_{0}=\pi_{i}^{\epsilon_{i}(u)}\left(u, \tilde{e}_{i} v\right)_{0}$ for $u, v \in \mathcal{L} / q \mathcal{L}$ (Here $\pi$ is understood as 1 or -1 for $\diamond \in\{+,-\}$, respectively).

(iii) $B$ is a $\pi$-orthonormal $\pi$-basis of $\mathcal{L} / q \mathcal{L}$ with respect to $(\cdot, \cdot)_{0}$.

(iv) $\mathcal{L}=\{u \in V ;(u, \mathcal{L}) \subseteq \mathcal{A}\}$.

Proof. For notational simplicity and certainty, we will prove the case (2) in detail, while the case (1) is entirely similar.

The same easy reduction as in the proof of [Ka, Proposition 5.1.1] reduces the proof of Parts (i) and (ii) to the verification of the identity

$$
\left(\tilde{f}_{i} u, v\right) \equiv \pi_{i}^{\epsilon_{i}(u)}\left(u, \tilde{e}_{i} v\right) \quad \bmod q \mathcal{A},
$$

where $u=F_{i}^{(n)} u_{0} \in \mathcal{L}(\lambda)_{\diamond}^{\lambda+\zeta+\alpha_{i}}, v=F_{i}^{(m)} v_{0} \in \mathcal{L}(\lambda)_{\diamond}^{\lambda+\zeta}$ with $E_{i} u_{0}=E_{i} v_{0}=0$.

To that end, we have the following computation (compare [Ka, (5.1.2)]):

$$
\begin{aligned}
\left(F_{i}^{(n+1)} u_{0}, F_{i}^{(m)} v_{0}\right) & =\delta_{n+1, m} q_{i}^{m\left\langle\alpha_{i}^{\vee}, \lambda+\zeta\right\rangle+m^{2}}\left(E_{i}^{(m)} F_{i}^{(m)} u_{0}, v_{0}\right) \\
& =\delta_{n+1, m} \pi_{i}^{m^{2}+\left(\begin{array}{c}
m+1 \\
2
\end{array}\right)} q_{i}^{m\left\langle\alpha_{i}^{\vee}, \lambda+\zeta\right\rangle+m^{2}}\left[\begin{array}{c}
\left\langle\alpha_{i}^{\vee}, \lambda+\zeta\right\rangle+2 m \\
m
\end{array}\right]\left(u_{0}, v_{0}\right) \\
& \equiv \delta_{n+1, m} \pi_{i}^{\left(\begin{array}{c}
m \\
2
\end{array}\right)}\left(u_{0}, v_{0}\right) \bmod q \mathcal{A},
\end{aligned}
$$

where we have used $\left(\begin{array}{c}m+1 \\ 2\end{array}\right)+m^{2} \equiv\left(\begin{array}{c}m \\ 2\end{array}\right) \bmod 2$. Therefore $\left(\tilde{f}_{i} u, v\right)_{0}=\pi_{i}^{m-1}\left(u, \tilde{e}_{i} v\right)_{0}$. Since $m-1=n=\epsilon_{i}(u)$, the identity (6.1) follows, and whence (i) and (ii).

Part (iii) follows by induction on weights and using Theorem 4.15 from the identity

$$
\left(b, b^{\prime}\right)_{0}=\left(\tilde{f}_{i} \tilde{e}_{i} b, b^{\prime}\right)_{0}=\left(\tilde{e}_{i} b, \tilde{e}_{i} b^{\prime}\right)_{0},
$$


where $b, b^{\prime} \in B$ and $i \in I$ is chosen such that $\tilde{e}_{i} b \in B$.

To prove (iv), it remains to verify that $\{u \in V ;(u, \mathcal{L}) \subseteq \mathcal{A}\} \subseteq \mathcal{L}$ thanks to (i). Denote $\operatorname{sgn}(b)=(b, b)_{0}$. Suppose $u \in V$ is a $\mu$-weight vector such that $(u, \mathcal{L}) \subseteq \mathcal{A}$. By Theorem 4.15 and the definition of crystal basis and $\pi$-basis, one can find $B_{\mu}^{0} \subset$ $B_{\mu}$ which is an honest basis for $\mathcal{L}_{\mu} / q \mathcal{L}_{\mu}$. Then $u$ can be written as $u=\sum_{b \in B_{\mu}^{0}} c_{b} u_{b}$. where $u_{b}+q \mathcal{L}=b$ and $c_{b} \in \mathbb{Q}(q)$. Assume $u \notin \mathcal{L}$. Then there exists a minimal $r \in \mathbb{Z}_{>0}$ such that $q^{r} c_{b} \in \mathcal{A}$ for all $b \in B_{\mu}$. Since $(u, \mathcal{L}) \subset \mathcal{A}$, we have in particular that $\left(u, \operatorname{sgn}(b) q^{r-1} u_{b}\right) \in \mathcal{A}$. On the other hand, since $\left(u_{b}, u_{b^{\prime}}\right) \in q \mathcal{A}$ for $b \neq b^{\prime}$, we compute that $\left(u, \operatorname{sgn}(b) q^{r-1} u_{b}\right) \in q^{r-1} c_{b}+\mathcal{A}$ for all $b$, whence $q^{r-1} c_{b} \in \mathcal{A}$ for all $b$, contradicting the minimality of $r$. This completes the proof of the proposition.

Remark 6.2. (1) One can formulate a version of Proposition 6.1 with $\diamond=\emptyset$ and the bilinear form $(\cdot, \cdot)_{0}$ taking value in $\mathbb{Q}^{\pi}$.

(2) In contrast to the usual quantum group setting in $\mathrm{Ka}, \mathrm{Lu} 2,(\cdot, \cdot)_{0}$ here is not positive definite in general, as it could happen that $(b, b)_{0}=\pi$ for some crystal basis element. In particular, the well-known characterization in the usual quantum group setting that an element $u$ lies in the crystal lattice if and only if $(u, u) \in \mathcal{A}$ fails in our super setting in general; see Example 6.3.

Example 6.3. Let $\mathbf{U}$ be the quantum osp $(1 \mid 4)$, with $\alpha_{1}$ the short root and $\alpha_{2}$ the long root. Then $\tilde{f}_{1}^{4} \tilde{f}_{2} \cdot 1=F_{1}^{(4)} F_{2}$ and $\tilde{f}_{1}^{3} \tilde{f}_{2} \tilde{f}_{1} \cdot 1=F_{1}^{(3)}\left(F_{2} F_{1}-q^{2} F_{1} F_{2}\right)+$ $q^{2} F_{1}^{(4)} F_{2}$ (these will be canonical basis elements as developed in Section 7). A direct computation shows that

$$
\left(F_{1}^{(3)}\left(F_{2} F_{1}-q^{2} F_{1} F_{2}\right), F_{1}^{(4)} F_{2}\right)=0,
$$

and also

$$
\begin{aligned}
& \left(F_{1}^{(4)} F_{2}, F_{1}^{(4)} F_{2}\right)=(\pi q)^{6}\left([4]^{!}\right)^{-1} \in 1+q^{2} \mathbb{Z}^{\pi}[[q]], \\
& \left(F_{1}^{(3)}\left(F_{2} F_{1}-q^{2} F_{1} F_{2}\right), F_{1}^{(3)}\left(F_{2} F_{1}-q^{2} F_{1} F_{2}\right)\right) \\
& =(\pi q)^{3}\left([3]^{!}\right)^{-1}\left(1-q^{4}\right) \in \pi+q^{2} \mathbb{Z}^{\pi}[[q]] .
\end{aligned}
$$

It follows that

(1) $\left(\tilde{f}_{1}^{4} \tilde{f}_{2} \cdot 1, \tilde{f}_{1}^{4} \tilde{f}_{2} \cdot 1\right)=1 \bmod q^{2} \mathbb{Z}^{\pi}[[q]] ;$

(2) $\left(\tilde{f}_{1}^{3} \tilde{f}_{2} \tilde{f}_{1} \cdot 1, \tilde{f}_{1}^{3} \tilde{f}_{2} \tilde{f}_{1} \cdot 1\right)=\pi \bmod q^{2} \mathbb{Z}^{\pi}[[q]] ;$

(3) $\left(\tilde{f}_{1}^{4} \tilde{f}_{2} \cdot 1, \tilde{f}_{1}^{3} \tilde{f}_{2} \tilde{f}_{1} \cdot 1\right)=q^{2} \bmod q^{4} \mathbb{Z}^{\pi}[[q]]$.

Now (1) and (2) provide us an example that the squared norm of (canonical basis) elements in $B(\infty)$ of the same weight do not have uniform sign. Combined with (3), this implies that $\mathcal{L}(\infty) \subsetneq\left\{u \in \mathbf{U}^{-} \mid(u, u) \in \mathcal{A}\right\}$, since $q^{-1}(1-\pi)\left(\tilde{f}_{1}^{4} \tilde{f}_{2} \cdot 1+\tilde{f}_{1}^{3} \tilde{f}_{2} \tilde{f}_{1} \cdot 1\right)$ belongs to the right-hand side, but not to $\mathcal{L}(\infty)$.

6.2. Polarization and $\varrho$. We simply formulate the counterpart of [Ka, $\S 5.2]$.

Recall from [CHW, §2.1.2] an algebra automorphism $\omega$ on $\mathbf{U}$ and an algebra anti-involution $\sigma$ on $\mathbf{U}$ (i.e., $\sigma(x y)=\sigma(y) \sigma(x)$ ). Note that $\sigma$ fixes each $E_{i}$ but not $F_{i}$ (a super phenomenon). In particular, $\varrho:=\omega \sigma \omega^{-1}$ is a (nonsuper) algebra 
anti-involution on $\mathbf{U}$ such that

$$
\varrho\left(F_{i}\right)=F_{i}, \quad \varrho\left(E_{i}\right)=\pi_{i} \widetilde{J}_{i} E_{i}, \quad \varrho\left(K_{\mu}\right)=K_{-\mu}, \quad \varrho\left(J_{\mu}\right)=J_{\mu} \quad\left(i \in I, \mu \in P^{\vee}\right) .
$$

We start with some lemmas.

Lemma 6.4. For $i, j \in I$ and $P, Q \in \mathbf{U}^{-}$, we have

$$
\begin{aligned}
\left(\operatorname{Ad}\left(\widetilde{K}_{i}\right) E_{i}^{\prime \prime}\right) \circ E_{j}^{\prime} & =\pi^{p(i) p(j)} E_{j}^{\prime} \circ\left(\operatorname{Ad}\left(\widetilde{K}_{i}\right) E_{i}^{\prime \prime}\right), \\
\left(P F_{i}, Q\right) & =\pi_{i}^{p(P)}\left(P, \operatorname{Ad}\left(\widetilde{K}_{i}\right) E_{i}^{\prime \prime} Q\right) .
\end{aligned}
$$

Proof. The identity (6.3) is a reformulation of the $q$-commutativity of $E_{i}^{\prime \prime}$ and $E_{j}^{\prime}$ in Proposition 3.5. Then (6.4) is proved in the same way as in [Ka Lemma 5.2.2], modified by (6.3) above.

We shall also write $\varrho(Q)$ as $Q^{\varrho}$ for $Q \in \mathbf{U}^{-}$.

Lemma 6.5. We have $\left(E_{i}^{\prime}\left(Q^{\varrho}\right)\right)^{\varrho}=\pi_{i}^{p(Q)+1} \operatorname{Ad}\left(\widetilde{K}_{i}\right) E_{i}^{\prime \prime} Q$, for $Q \in \mathbf{U}^{-}$.

Proof. We can rewrite (3.6) as

$$
\left[E_{i}, Q\right]=\frac{\widetilde{J}_{i}\left(\operatorname{Ad}\left(\widetilde{K}_{i}\right) E_{i}^{\prime \prime} Q\right) \widetilde{K}_{i}-\left(\left(\operatorname{Ad} \widetilde{K}_{i}^{-1}\right) E_{i}^{\prime} Q\right) \widetilde{K}_{i}^{-1}}{\pi_{i} q_{i}-q_{i}^{-1}} .
$$

Applying $\varrho$ (see (6.2) $)$ to the above identity gives us

$$
\left[Q^{\varrho}, E_{i}^{\varrho}\right]=\frac{\widetilde{K}_{i}^{-1} \widetilde{J}_{i}\left(\operatorname{Ad}\left(\widetilde{K}_{i}\right) E_{i}^{\prime \prime} Q\right)^{\varrho}-\widetilde{K}_{i}\left(\left(\operatorname{Ad} \widetilde{K}_{i}^{-1}\right) E_{i}^{\prime} Q\right)^{\varrho}}{\pi_{i} q_{i}-q_{i}^{-1}} .
$$

Using $\left[Q^{\varrho}, E_{i}^{\varrho}\right]=-\pi_{i}^{p(Q)}\left[\pi_{i} \widetilde{J}_{i} E_{i}, Q^{\varrho}\right]$ and noting $\widetilde{J}_{i}$ is central, we rewrite the above as

$$
\left[E_{i}, Q^{\varrho}\right]=\pi_{i}^{p(Q)+1} \frac{\widetilde{J}_{i} \widetilde{K}_{i}\left(\left(\operatorname{Ad} \widetilde{K}_{i}^{-1}\right) E_{i}^{\prime} Q\right)^{\varrho}-\widetilde{K}_{i}^{-1}\left(\operatorname{Ad}\left(\widetilde{K}_{i}\right) E_{i}^{\prime \prime} Q\right)^{\varrho}}{\pi_{i} q_{i}-q_{i}^{-1}} .
$$

A comparison with (3.6) (by setting $y=Q^{\varrho}$ ) gives us the desired formula.

Now we are ready to show that $\varrho$ is an isometry (without signs).

Proposition 6.6. For $P, Q \in \mathbf{U}^{-}$, we have

$$
\left(P^{\varrho}, Q^{\varrho}\right)=(P, Q) \text {. }
$$

Proof. The claim is trivial when $P=1$, and so it suffices to prove that the identity (6.5) implies that

$$
\left(\left(P F_{i}\right)^{\varrho}, Q^{\varrho}\right)=\left(P F_{i}, Q\right)
$$

We will assume without loss of generality that $p(Q)=p\left(P F_{i}\right)=p(P)+1$, as otherwise both sides are clearly equal to 0 :

$$
\begin{aligned}
\left(\left(P F_{i}\right)^{\varrho}, Q^{\varrho}\right) & =\left(F_{i} P^{\varrho}, Q^{\varrho}\right)=\left(P^{\varrho}, E_{i}^{\prime} Q^{\varrho}\right) \\
& =\left(P,\left(E_{i}^{\prime} Q^{\varrho}\right)^{\varrho}\right)=\pi_{i}^{p(Q)+1}\left(P, \operatorname{Ad}\left(\widetilde{K}_{i}\right) E_{i}^{\prime \prime} Q\right) \\
& =\pi_{i}^{p(Q)+1} \pi_{i}^{p(P)}\left(P F_{i}, Q\right)=\left(P F_{i}, Q\right) .
\end{aligned}
$$

This completes the proof. 
The fact that $\varrho\left(\mathcal{L}(\infty)_{+}\right)=\mathcal{L}(\infty)_{+}$follows easily from (6.5) and the orthonormality characterization of crystal lattice in the standard quantum group setting Lu1, Lu2, Ka. While such orthonormality characterization fails in our super setting as noted in Remark 6.2 (2), the $\varrho$-stable property of the crystal lattice remains to be true.

Proposition 6.7. For $\diamond \in\{\emptyset,-\}$, we have $\varrho\left(\mathcal{L}(\infty)_{\diamond}\right)=\mathcal{L}(\infty)_{\diamond}$.

Remark 6.8. Proposition [6.7 is proved in [CFLW, Proposition 3.4], as it is an immediate consequence of connections between $\mathbf{U}$ and the Drinfeld-Jimbo quantum groups developed therein.

6.3. A comparison of polarizations. Now let us compare the bilinear forms on $\mathcal{L}(\lambda)$ and $\mathcal{L}(\infty)$.

Proposition 6.9. For given $x, y \in \mathcal{L}(\infty)_{\zeta}$, take $\lambda \gg 0$. Then $\left(x v_{\lambda}^{+}, y v_{\lambda}^{+}\right)_{0}=$ $(x, y)_{0}$.

Proof. This is obvious for $\zeta=0$. We proceed by induction on the height of $\zeta$. We can write $x=F_{i} x^{\prime}$ for some $i \in I$. Then

$$
\begin{aligned}
\left(x v_{\lambda}^{+}, y v_{\lambda}^{+}\right) & =\left(x^{\prime} v_{\lambda}^{+}, q_{i}^{-1} \widetilde{K}_{i} E_{i} y v_{\lambda}^{+}\right) \\
& =\left(x^{\prime} v_{\lambda}^{+}, \frac{\widetilde{J}_{i} \widetilde{K}_{i}^{2} E_{i}^{\prime \prime}(y)-E_{i}^{\prime}(y)}{\pi_{i} q_{i}^{2}-1} v_{\lambda}^{+}\right) \\
& =\frac{\left(\pi_{i} q_{i}^{2}\right)\left\langle\alpha_{i}^{\vee}, \lambda+|y|+\alpha_{i}\right\rangle}{\pi q_{i}^{2}-1}\left(x^{\prime} v_{\lambda}^{+}, E_{i}^{\prime \prime}(y) v_{\lambda}^{+}\right)+\frac{1}{1-\pi_{i} q_{i}^{2}}\left(x^{\prime} v_{\lambda}^{+}, E_{i}^{\prime}(y) v_{\lambda}^{+}\right) .
\end{aligned}
$$

Hence, by induction and the assumption $\lambda \gg 0$, we have

$$
\left(x v_{\lambda}^{+}, y v_{\lambda}^{+}\right)_{0}=\left(x^{\prime} v_{\lambda}^{+}, E_{i}^{\prime}(y) v_{\lambda}^{+}\right)_{0}=\left(x^{\prime}, E_{i}^{\prime}(y)\right)_{0}=(x, y)_{0} .
$$

The proposition is proved.

We can also relate these bilinear forms to the bilinear form on $\mathbf{U}^{-}$given in CHW]. We recall from that paper the following notation. The algebra $\mathbf{f}$ is the $\mathbb{Q}(q)^{\pi}$-algebra generated by $\theta_{i}$ such that there is an isomorphism $\mathbf{f} \cong \mathbf{U}^{-}$given by $\theta_{i} \mapsto \theta_{i}^{-}=F_{i}$. Via this identification, the twisted derivations $r_{i}$ and ${ }_{i} r$ on $\mathbf{f}$ lead to twisted derivation on $\mathbf{U}^{-}$denoted by the same notations. There is a bilinear form $(\cdot, \cdot)$ on $\mathbf{f}$ and hence on $\mathbf{U}^{-}$CHW, $\left.\S 1\right]$ which will be denoted by $(\cdot, \cdot)_{L}$ here to avoid conflict of notation, and its bar conjugate $\{\cdot, \cdot\}$ defined by $\{x, y\}=\overline{(\bar{x}, \bar{y})_{L}}$, for $x, y \in \mathbf{U}^{-}$.

By comparing the formula for $E_{i}^{\prime}$ in Lemma 3.4 and the formula for $r_{i}$ in $\mathrm{CHW}$, $\S 1.3 .13]$, we have by

$$
E_{i}^{\prime}(y)=\pi_{i}^{p(y)-p(i)} q_{i}^{\left\langle\alpha_{i}^{\vee},|y|+\alpha_{i}\right\rangle} r_{i}(y), \quad \text { for } y \in \mathbf{U}^{-} .
$$

By [CHW, Lemma 1.3.14], $r_{i}(y)=\pi_{i}^{p(y)-p(i)} q_{i}^{\left\langle\alpha_{i}^{\vee},-|y|-\alpha_{i}\right\rangle} \overline{{ }_{i} r(\bar{y})}$, and hence we obtain

$$
E_{i}^{\prime}(y)=\overline{{ }_{i} r(\bar{y})} \text {. }
$$


Proposition 6.10. Let $x, y \in \mathbf{U}^{-}$. Then $\lim _{\lambda \mapsto \infty}\left(x^{-} v_{\lambda}^{+}, y^{-} v_{\lambda}^{+}\right)=\{x, y\}$ in the q-adic norm.

Proof. Recall [CHW, §1] a defining property of $(\cdot, \cdot)_{L}$ is that

$$
\left(\theta_{i}, \theta_{i}\right)_{L}\left(x,{ }_{i} r(y)\right)_{L}=\left(\theta_{i} x, \theta_{i} y\right)_{L} .
$$

We have $\left(\pi_{i} q_{i}^{2}\right)^{\left\langle\alpha_{i}^{\vee}, \lambda+|y|+\alpha_{i}\right\rangle} \rightarrow 0$ in the $q$-adic norm as $\lambda \mapsto+\infty$; so using the computations in the proof of Proposition 6.9, we find that

$$
\begin{aligned}
\lim _{\lambda \mapsto \infty}\left(x v_{\lambda}^{+}, y v_{\lambda}^{+}\right) & =\frac{1}{1-\pi_{i} q_{i}^{2}}\left\{x^{\prime}, E_{i}^{\prime}(y)\right\}=\left\{\theta_{i}, \theta_{i}\right\}\left\{x^{\prime}, \overline{{ }_{i} r(\bar{y})}\right\} \\
& =\overline{\left(\theta_{i}, \theta_{i}\right)_{L}\left(\overline{x^{\prime}},{ }_{i} r(\bar{y})\right)_{L}}=\overline{\left(\theta_{i} \overline{x^{\prime}}, \bar{y}\right)_{L}}=\{x, y\} .
\end{aligned}
$$

The proposition is proved.

\section{Canonical bases}

In this section we shall prove the existence of canonical bases (= global crystal bases) for $\mathbf{U}^{-}$and all integrable modules.

7.1. The integral form of $\mathbf{U}$. Let ${ }_{\mathbb{Z}} \mathbf{U}$ be the $\mathbb{Z}\left[q, q^{-1}\right]$-subalgebra of $\mathbf{U}$ generated by $F_{i}^{(n)}, E_{i}^{(n)}, J_{\mu}, K_{\mu}^{ \pm 1}$ and $\left[\begin{array}{c}K_{i} ; 0 \\ a\end{array}\right]$ for all $i \in I, n \in \mathbb{Z}_{\geq 0}$, and $a \in \mathbb{Z}$. We set ${ }_{\mathbb{Z}} \mathbf{U}^{-}$ to be the $\mathbb{Z}\left[q, q^{-1}\right]$-subalgebra generated by $F_{i}^{(n)}$ for $i \in I$ and $n \in \mathbb{Z}_{\geq 0}$. Then ${ }_{\mathbb{Z}} \mathbf{U}$ and ${ }_{\mathbb{Z}} \mathbf{U}^{-}$are stable under the bar involution (2.1). Moreover, ${ }_{\mathbb{Z}} \mathbf{U}^{-}$is stable under $E_{i}^{\prime}$ whence $\mathbb{Z}_{\mathbb{Z}} \mathbf{U}^{-}$is stable under Kashiwara operators $\tilde{e}_{i}$ and $\tilde{f}_{i}$; therefore

$$
u=\sum_{n \geq 0} F_{i}^{(n)} u_{n} \in{ }_{\mathbb{Z}} \mathbf{U}^{-} \text {and } e_{i}^{\prime} u_{n}=0 \Longrightarrow u_{n} \in{ }_{\mathbb{Z}} \mathbf{U}^{-} .
$$

Let $\left(F_{i}^{n} \mathbf{U}^{-}\right)_{\mathbb{Z}}=F_{i}^{n} \mathbf{U}^{-} \cap_{\mathbb{Z}} \mathbf{U}^{-}$. Then

$$
\left(F_{i}^{n} \mathbf{U}^{-}\right)_{\mathbb{Z}}=\sum_{k \geq n} F_{i}^{(k)}{ }_{\mathbb{Z}} \mathbf{U}^{-}, \quad \text { for } n \geq 0 .
$$

Moreover, $u=\sum F_{i}^{(k)} u_{k} \in\left(F_{i}^{n} \mathbf{U}^{-}\right)_{\mathbb{Z}}$ if and only if $u_{k}=0$ for $k<n$. Set $\mathcal{L}_{\mathbb{Z}}(\infty)=$ $\mathcal{L}(\infty) \cap{ }_{\mathbb{Z}} \mathbf{U}^{-}$. Then $\mathcal{L}_{\mathbb{Z}}(\infty)$ is stable under the Kashiwara operators $\tilde{e}_{i}$ and $\tilde{f}_{i}$. Therefore, $B(\infty) \subset \mathcal{L}_{\mathbb{Z}}(\infty) / q \mathcal{L}_{\mathbb{Z}}(\infty) \subset \mathcal{L}(\infty) / q \mathcal{L}(\infty)$.

Similarly, for $\diamond \in\{+,-\}$, we can define the integral forms $\mathbb{Z}_{\diamond} \mathbf{U}_{\diamond}$ and $\mathbb{Z}_{\mathbb{Z}} \mathbf{U}_{\diamond}^{-}$of the specializations $\mathbf{U}_{\diamond}$ and $\mathbf{U}_{\diamond}^{-}$, respectively. Then $\mathcal{L}_{\mathbb{Z}}(\infty)_{\diamond}:=\mathcal{L}(\infty)_{\diamond} \cap_{\mathbb{Z}} \mathbf{U}_{\diamond}^{-}$is stable under Kashiwara operators.

Let $\mathcal{A}_{\mathbb{Z}}$ be the $\mathbb{Z}^{\pi}$-subalgebra of $\mathbb{Q}(q)^{\pi}$ generated by $q$ and $\left(1-\left(\pi q^{2}\right)^{n}\right)^{-1}$ for $n \geq 1$. Similarly, for $\diamond=+,-$, let $\mathcal{A}_{\mathbb{Z}}^{\diamond}$ be the $\mathbb{Z}$-subalgebra of $\mathbb{Q}(q)^{\pi}$ generated by $q$ and $\left(1-\left(\diamond q^{2}\right)^{n}\right)^{-1}$ for $n \geq 1$. Letting $K_{\mathbb{Z}}^{\diamond}$ be the subalgebra generated by $\mathcal{A}_{\mathbb{Z}}^{\diamond}$ and $q^{-1}$, we have $\mathcal{A}_{\mathbb{Z}}^{\diamond}=\mathcal{A} \cap K_{\mathbb{Z}}^{\diamond}$, for $\diamond \in\{\emptyset,+,-\}$. (As before, the superscript or subscript $\emptyset$ is dropped by convention.) Then we see that $\left({ }_{\mathbb{Z}} \mathbf{U}_{\diamond}^{-},{ }_{\mathbb{Z}} \mathbf{U}_{\diamond}^{-}\right) \subset K_{\mathbb{Z}}^{\diamond}$, whence $\left(\mathcal{L}_{\mathbb{Z}}(\infty)_{\diamond}, \mathcal{L}_{\mathbb{Z}}(\infty)_{\diamond}\right) \subset \mathcal{A}_{\mathbb{Z}}^{\diamond}$. Therefore, $(\cdot, \cdot)_{0}$ is $\mathbb{Z}^{\pi}$-valued on $\mathcal{L}_{\mathbb{Z}}(\infty) / q \mathcal{L}_{\mathbb{Z}}(\infty)$, and $\mathcal{L}_{\mathbb{Z}}(\infty) / q \mathcal{L}_{\mathbb{Z}}(\infty)$ is a free $\mathbb{Z}^{\pi}$-module with $\pi$-basis $B(\infty)$. Similarly, for $\diamond=+,-$, $(\cdot, \cdot)_{0}$ is $\mathbb{Z}$-valued on $\mathcal{L}_{\mathbb{Z}}(\infty)_{\diamond} / q \mathcal{L}_{\mathbb{Z}}(\infty)_{\diamond}$, and $\mathcal{L}_{\mathbb{Z}}(\infty)_{\diamond} / q \mathcal{L}_{\mathbb{Z}}(\infty)_{\diamond}$ is a free $\mathbb{Z}$-module with $\pi$-basis $B(\infty)_{\diamond}$. 
7.2. The integral form of $V(\lambda)$. Let $\diamond \in\{\emptyset,+,-\}$. Set $V_{\mathbb{Z}}(\lambda)_{\diamond}={ }_{\mathbb{Z}} \mathbf{U}_{\diamond}^{-} v_{\lambda}^{+}$. Then $V_{\mathbb{Z}}(\lambda)_{\diamond}$ is a ${ }_{\mathbb{Z}} \mathbf{U}_{\diamond}$-module. We set also, for $n \geq 0$,

$$
\left(F_{i}^{n} V(\lambda)_{\diamond}\right)_{\mathbb{Z}}=\left(F_{i}^{n} \mathbf{U}_{\diamond}^{-}\right)_{\mathbb{Z}} v_{\lambda}^{+}=\sum_{k \geq n} F_{i}^{(k)} V_{\mathbb{Z}}(\lambda)_{\diamond}
$$

Note that $V_{\mathbb{Z}}(\lambda)_{\diamond}$ and $\left(F_{i}^{n} V(\lambda)_{\diamond}\right)_{\mathbb{Z}}$ are bar-invariant.

Let $\mathcal{L}_{\mathbb{Z}}(\lambda)_{\diamond}=V_{\mathbb{Z}}(\lambda)_{\diamond} \cap \mathcal{L}(\lambda)_{\diamond}$. Since $\mathcal{L}(\lambda)_{\diamond}=\wp_{\lambda}\left(\mathcal{L}(\infty)_{\diamond}\right)$ we have

$$
\mathcal{L}_{\mathbb{Z}}(\lambda)_{\diamond} \subset \wp_{\lambda}\left(\mathcal{L}_{\mathbb{Z}}(\infty)_{\diamond}\right)
$$

and so $B(\lambda)_{\diamond} \subset \mathcal{L}_{\mathbb{Z}}(\lambda)_{\diamond} / q \mathcal{L}_{\mathbb{Z}}(\lambda)_{\diamond} \subset \mathcal{L}(\lambda)_{\diamond} / q \mathcal{L}(\lambda)_{\diamond}$.

The following is a $\pi$-analogue of [Ka, Lemma 6.1.14].

Lemma 7.1. Let $\lambda \in P, i \in I$, and $u \in M^{\lambda}$ for an integrable $\mathbf{U}$-module $M$. Assume $n=-\left\langle\alpha_{i}^{\vee}, \lambda\right\rangle \geq 1$. Then we have

$$
u=\pi_{i}^{\left(\begin{array}{c}
n \\
2
\end{array}\right)} \sum_{k \geq n}(-1)^{k-n}\left[\begin{array}{l}
k-1 \\
k-n
\end{array}\right]_{i} F_{i}^{(k)} E_{i}^{(k)} u .
$$

Proof. We may assume $u=F_{i}^{(m)} v$ with $v \in \operatorname{ker} e_{i} \cap M_{\lambda+m \alpha_{i}}$ with $m \geq n$. Then

$$
\begin{aligned}
\sum_{k \geq n}(-1)^{k-n} & {\left[\begin{array}{l}
k-1 \\
k-n
\end{array}\right]_{i} F_{i}^{(k)} E_{i}^{(k)} u } \\
& =\sum_{k \geq n}(-1)^{k-n}\left[\begin{array}{c}
k-1 \\
k-n
\end{array}\right]_{i} F_{i}^{(k)} E_{i}^{(k)} F_{i}^{(m)} v \\
& =\sum_{m \geq k \geq n} \pi_{i}^{k m+\left(\begin{array}{c}
k+1 \\
2
\end{array}\right)}(-1)^{k-n}\left[\begin{array}{c}
k-1 \\
k-n
\end{array}\right]_{i}\left[\begin{array}{c}
k+m-n \\
k
\end{array}\right]_{i} F_{i}^{(k)} F_{i}^{(m-k)} v \\
& =\sum_{m \geq k \geq n} \pi_{i}^{k m+\left(\begin{array}{c}
k+1 \\
2
\end{array}\right)}(-1)^{k-n}\left[\begin{array}{c}
k-1 \\
k-n
\end{array}\right]_{i}\left[\begin{array}{c}
k+m-n \\
k
\end{array}\right]_{i}\left[\begin{array}{c}
m \\
k
\end{array}\right]_{i} \cdot F_{i}^{(m)} v
\end{aligned}
$$

By a change of variables with $t=k-n$ and $r=m-n$, we have

$$
\begin{aligned}
& \sum_{m \geq k \geq n} \pi_{i}^{k m+\left(\begin{array}{c}
k+1 \\
2
\end{array}\right)}(-1)^{k-n}\left[\begin{array}{c}
k-1 \\
k-n
\end{array}\right]_{i}\left[\begin{array}{c}
k+m-n \\
k
\end{array}\right]_{i}\left[\begin{array}{c}
m \\
k
\end{array}\right]_{i} \\
= & \sum_{t=0}^{r}(-1)^{t} \pi_{i}^{(t+n)(r+n)+\left(\begin{array}{c}
t+n+1 \\
2
\end{array}\right)}\left[\begin{array}{c}
t+n-1 \\
t
\end{array}\right]_{i}\left[\begin{array}{c}
t+r+n \\
r
\end{array}\right]_{i}\left[\begin{array}{l}
r+n \\
t+n
\end{array}\right]_{i} .
\end{aligned}
$$

The proof is completed by [Ka, (6.1.19)] if $i \in I_{0}$ and by the following lemma if $i \in I_{1}$.

Lemma 7.2. We have the following identity for $r \geq 0$ and $n \geq 1$ :

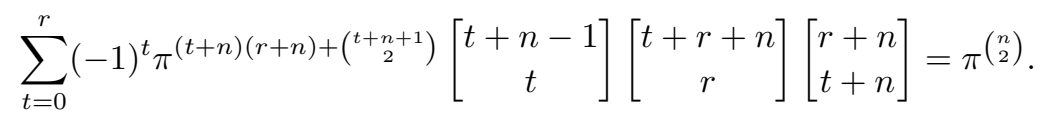

Proof. We first introduce the following notation. Let

$$
\langle n\rangle:=\left.[n]\right|_{\pi=1}=\frac{q^{n}-q^{-n}}{q-q^{-1}}, \quad\{n\}_{x}:=\frac{1-x^{n}}{1-x} .
$$


We extend this notation to factorials and binomials in a self-explanatory manner. We also note that

$$
\begin{aligned}
& {[n]=(\pi q)^{n-1}\{n\}_{\pi q^{-2}},} \\
& \langle n\rangle=q^{n-1}\{n\}_{q^{-2}} .
\end{aligned}
$$

The identity [Ka, (6.1.19)] may be stated in this notation as

$$
\sum_{t=0}^{r}(-1)^{t}\left\langle\begin{array}{c}
t+n-1 \\
t
\end{array}\right\rangle\left\langle\begin{array}{c}
t+r+n \\
r
\end{array}\right\rangle\left\langle\begin{array}{l}
r+n \\
t+n
\end{array}\right\rangle=1
$$

which can be transformed using (7.2) into

$$
\sum_{t=0}^{r}(-1)^{t}\left(q^{-2}\right)\left(\begin{array}{c}
t+1 \\
2
\end{array}\right)-(n+t) r\left\{\begin{array}{c}
t+n-1 \\
t
\end{array}\right\}_{q^{-2}}\left\{\begin{array}{c}
t+r+n \\
r
\end{array}\right\}_{q^{-2}}\left\{\begin{array}{l}
r+n \\
t+n
\end{array}\right\}_{q^{-2}}=1 .
$$

Note that this is a general identity for the $\{n\}_{x}$, so

$$
\sum_{t=0}^{r}(-1)^{t} x^{\left(\begin{array}{c}
t+1 \\
2
\end{array}\right)-(n+t) r}\left\{\begin{array}{c}
t+n-1 \\
t
\end{array}\right\}_{x}\left\{\begin{array}{c}
t+r+n \\
r
\end{array}\right\}_{x}\left\{\begin{array}{l}
r+n \\
t+n
\end{array}\right\}_{x}=1 .
$$

In particular, setting $x=\pi q^{-2}$ and using (7.1), we obtain

$$
\sum_{t=0}^{r}(-1)^{t} \pi^{\left(\begin{array}{c}
t+1 \\
2
\end{array}\right)-(n+t) r}\left[\begin{array}{c}
t+n-1 \\
t
\end{array}\right]\left[\begin{array}{c}
t+r+n \\
r
\end{array}\right]\left[\begin{array}{c}
r+n \\
t+n
\end{array}\right]=1 .
$$

The lemma follows since $\left(\begin{array}{c}t+1 \\ 2\end{array}\right)-(n+t) r=(t+n)(r+n)+\left(\begin{array}{c}t+n+1 \\ 2\end{array}\right)+\left(\begin{array}{c}n \\ 2\end{array}\right) \bmod 2$.

Proposition 7.3. Let $M$ be an integrable $\mathbf{U}$-module and $M_{\mathbb{Z}} a_{\mathbb{Z}} \mathbf{U}$-weight submodule of $M$. Let $\lambda \in P$ and $i \in I$. Suppose $n=-\left\langle\alpha_{i}^{\vee}, \lambda\right\rangle \geq 0$. Then

$$
\left(M_{\mathbb{Z}}\right)^{\lambda}=\sum_{k \geq n} F_{i}^{(k)}\left(M_{\mathbb{Z}}\right)^{\lambda+k \alpha_{i}}
$$

Proof. The lemma implies that $\left(M_{\mathbb{Z}}\right)^{\lambda} \subseteq \sum_{k \geq n} F_{i}^{(k)}\left(M_{\mathbb{Z}}\right)^{\lambda+k \alpha_{i}}$. The reverse inclusion is clear.

7.3. Existence of canonical bases. Let us consider the following collection $\left(G_{l}\right)$ of statements for $l \geq 0$, where $\diamond \in\{\emptyset,+,-\}$.

$\left(G_{l} .1\right)$ For any $\zeta \in Q^{-}(l)$,

$$
\left({ }_{\mathbb{Z}} \mathbf{U}_{\diamond}^{-}\right)^{\zeta} \cap \mathcal{L}_{\mathbb{Z}}(\infty)_{\diamond} \cap \overline{\mathcal{L}_{\mathbb{Z}}(\infty)_{\diamond}} \rightarrow \mathcal{L}_{\mathbb{Z}}(\infty)_{\diamond}^{\zeta} / q \mathcal{L}_{\mathbb{Z}}(\infty)_{\diamond}^{\zeta}
$$

is an isomorphism.

$\left(G_{l} .2\right)$ For any $\zeta \in Q^{-}(l)$,

$$
V_{\mathbb{Z}}^{-}(\lambda)_{\diamond}^{\lambda+\zeta} \cap \mathcal{L}_{\mathbb{Z}}(\lambda)_{\diamond} \cap \overline{\mathcal{L}_{\mathbb{Z}}(\lambda)_{\diamond}} \rightarrow \mathcal{L}_{\mathbb{Z}}(\lambda)_{\diamond}^{\lambda+\zeta} / q \mathcal{L}_{\mathbb{Z}}(\lambda)_{\diamond}^{\lambda+\zeta}
$$

is an isomorphism.

Let $G, G_{\lambda}$ be the inverses of these isomorphisms.

$\left(G_{l} .3\right)$ For any $\zeta \in Q^{-}(l), n \geq 0$, and $b \in \tilde{f}_{i}^{n}\left(B(\infty)_{\diamond}^{\zeta+n \alpha_{i}}\right)$,

$$
G(b) \in f_{i}^{n} \mathbf{U}^{-} .
$$


The case $\diamond=+$ is as in [Ka, $\S 6-7]$, and so let us now consider the case $\diamond=-$.

Note that when $l=0$, these statements are obvious. We shall prove $G_{l}$ by induction on $l$, so assume $l>0$ and $G_{l-1}$ holds.

Lemma 7.4. For $\zeta \in Q^{-}(l-1)$ we have

$$
\begin{aligned}
\left({ }_{\mathbb{Z}} \mathbf{U}_{-}^{-}\right)^{\zeta} \cap \mathcal{L}_{\mathbb{Z}}(\infty)_{-} & =\bigoplus_{b \in B(\infty)_{-}^{\zeta}} \mathbb{Z}[q] G(b), \\
\left({ }_{\mathbb{Z}} \mathbf{U}_{-}^{-}\right)^{\zeta} & =\bigoplus_{b \in B(\infty)_{-}^{\zeta}} \mathbb{Z}\left[q, q^{-1}\right] G(b), \\
V_{\mathbb{Z}}(\lambda)_{-}^{\lambda+\zeta} \cap \mathcal{L}(\lambda)_{-} & =\bigoplus_{b \in B(\lambda)_{-}^{\lambda+\zeta}} \mathbb{Z}[q] G_{\lambda}(b), \\
V_{\mathbb{Z}}(\lambda)_{-}^{\lambda+\zeta} & =\bigoplus_{b \in B(\lambda)_{-}^{\lambda+\zeta}} \mathbb{Z}\left[q, q^{-1}\right] G_{\lambda}(b) .
\end{aligned}
$$

Proof. This follows from [Ka, Lemma 7.1.1] and $\left(G_{l-1} .1\right)-\left(G_{l-1} .2\right)$.

Lemma 7.5. For $\zeta \in Q^{-}(l-1), b \in \mathcal{L}_{\mathbb{Z}}(\infty)_{-} / q \mathcal{L}_{\mathbb{Z}}(\infty)_{-}$, and $\lambda \in P^{+}$,

$$
G(b) v_{\lambda}^{+}=G_{\lambda}\left(\hat{\wp}_{\lambda} b\right) \text {. }
$$

Lemma 7.6. For $\zeta \in Q^{-}(l-1)$ and $b \in \mathcal{L}_{\mathbb{Z}}(\infty)_{-} / q \mathcal{L}_{\mathbb{Z}}(\infty)_{-}$, we have

$$
\overline{G(b)}=G(b) \text {. }
$$

Proof. Set $Q=\frac{G(b)-\overline{G(b)}}{\pi q-q^{-1}}$. Then $Q \in\left({ }_{\mathbb{Z}} \mathbf{U}_{-}^{-}\right)^{\zeta} \cap q \mathcal{L}_{\mathbb{Z}}(\infty)_{-} \cap \overline{\mathcal{L}_{\mathbb{Z}}(\infty)_{-}}$, and hence $Q=0$.

Note that a super counterpart of the proof of [Ka, Lemma 7.5.1] that " $G_{i}(b)=$ $G_{j}(b)$ " requires the validity of Proposition 6.7. The remaining components of the inductive proof of $\left(G_{l} .1\right)-\left(G_{l} .3\right)$ proceed just as in [Ka, $\left.\S 7.4-\S 7.5\right]$.

Now since $\left(G_{l} .1\right)-\left(G_{l} .3\right)$ hold in the cases $\diamond \in\{+,-\}$, we can lift them to the level of a formal parameter $\pi$, and so $\left(G_{l} .1\right)-\left(G_{l} .3\right)$ for $\diamond=\emptyset$ also hold. Alternatively, one could go through Kashiwara's arguments in the setting of $\diamond=\emptyset$ directly.

We summarize the main theorem on canonical bases.

Theorem 7.7. Let $\diamond \in\{\emptyset,+,-\}$. Then

(1) $\{G(b) \mid b \in B(\infty)\}$ forms a bar-invariant $\pi$-basis for ${ }_{\mathbb{Z}} \mathbf{U}_{\diamond}^{-}$.

(2) For every $\lambda \in P^{+}, G(b) v_{\lambda}^{+}=G_{\lambda}\left(\hat{\wp}_{\lambda} b\right)$. Moreover, $\left\{G_{\lambda}(b) \mid b \in B(\lambda)\right\}$ forms a bar-invariant $\pi$-basis for $V_{\mathbb{Z}}(\lambda)_{\diamond}$.

The maximal variants $\{G(b)\} \cup\{\pi G(b)\}$ and $\left\{G_{\lambda}(b)\right\} \cup\left\{\pi G_{\lambda}(b)\right\}$ of the bases in the above theorem would be a signed basis in a more conventional sense. The bases in the above theorem (or these maximal variants) will be referred to as canonical bases.

Our canonical basis is a $\pi$-basis in the sense of Definition 3.14, but not a genuine basis in general. We do not regard this as a defect of our construction though as this is completely natural from the viewpoint of categorification $([\mathrm{HW}): \pi$ corresponds 
to "spin" (i.e., a parity shift functor $\Pi$ ), each (projective) indecomposable module $M$ comes from two "spin states" $\{M, \Pi M\}$, and there is no preferred choice among $M$ and $\Pi M$ a priori.

Example 7.8. Assume that $I_{1}$ contains $i, j$ such that $a_{i j}=a_{j i}=0$. Then $F_{i} F_{j}=$ $\pi F_{j} F_{i}$. Both $F_{i} F_{j}$ and $F_{j} F_{i}$ are canonical basis elements in $\mathbf{U}^{-}$, and there is no preferred choice among the two.

\section{REFERENCES}

[BKK] Georgia Benkart, Seok-Jin Kang, and Masaki Kashiwara, Crystal bases for the quantum superalgebra $U_{q}(\mathfrak{g l}(m, n))$, J. Amer. Math. Soc. 13 (2000), no. 2, 295-331, DOI 10.1090/S0894-0347-00-00321-0. MR:1694051 (2000m:17015)

[BKM] Georgia Benkart, Seok-Jin Kang, and Duncan Melville, Quantized enveloping algebras for Borcherds superalgebras, Trans. Amer. Math. Soc. 350 (1998), no. 8, 3297-3319, DOI 10.1090/S0002-9947-98-02058-3. MR.1451594 (99f:17014)

[CFLW] S. Clark, Z. Fan, Y. Li and W. Wang, Quantum supergroups III. Twistors, Commun. Math. Phys. 322 (2014), 415-436. MR3253707

[CHW] Sean Clark, David Hill, and Weiqiang Wang, Quantum supergroups I. Foundations, Transform. Groups 18 (2013), no. 4, 1019-1053, DOI 10.1007/s00031-013-9247-4. MR.3127986

[CW] Sean Clark and Weiqiang Wang, Canonical basis for quantum osp(1|2), Lett. Math. Phys. 103 (2013), no. 2, 207-231, DOI 10.1007/s11005-012-0592-3. MR3010460

[EKL] Alexander P. Ellis, Mikhail Khovanov, and Aaron D. Lauda, The odd nilHecke algebra and its diagrammatics, Int. Math. Res. Not. IMRN 4 (2014), 991-1062. MR3168401

[ELa] A. Ellis and A. Lauda, An odd categorification of $U_{q}\left(\mathfrak{s l}_{2}\right)$, arXiv:1307.7816.

[FLi] Z. Fan and Y. Li, Two-parameter quantum algebras, canonical bases and categorifications, IMRN (to appear), arXiv:1303.2429.

[HW] D. Hill and W. Wang, Categorification of quantum Kac-Moody superalgebras, Trans. Amer. Math. Soc. (to appear), arXiv:1202.2769v2.

[Jeo] Kyeonghoon Jeong, Crystal bases for Kac-Moody superalgebras, J. Algebra 237 (2001), no. 2, 562-590, DOI 10.1006/jabr.2000.8590. MR:1816704(2001m:17016)

[KKO] Seok-Jin Kang, Masaki Kashiwara, and Se-jin Oh, Supercategorification of quantum Kac-Moody algebras, Adv. Math. 242 (2013), 116-162, DOI 10.1016/j.aim.2013.04.008. MR.3055990

[KKT] S.-J. Kang, M. Kashiwara and S. Tsuchioka, Quiver Hecke superalgebras, arXiv:1107.1039.

[Ka] M. Kashiwara, On crystal bases of the Q-analogue of universal enveloping algebras, Duke Math. J. 63 (1991), no. 2, 465-516, DOI 10.1215/S0012-7094-91-06321-0. MR1115118 (93b:17045)

[Kac] V. G. Kac, Infinite-dimensional algebras, Dedekind's $\eta$-function, classical Möbius function and the very strange formula, Adv. in Math. 30 (1978), no. 2, 85-136, DOI 10.1016/0001-8708(78)90033-6. MR513845 (83a:17014a)

[Kw] J.-H. Kwon, Super duality and crystal bases for quantum orthosymplectic superalgebras, arXiv:1301.1756.

[Lu1] G. Lusztig, Canonical bases arising from quantized enveloping algebras, J. Amer. Math. Soc. 3 (1990), no. 2, 447-498, DOI 10.2307/1990961. MR1035415(90m:17023)

[Lu2] George Lusztig, Introduction to Quantum Groups, Progress in Mathematics, vol. 110, Birkhäuser Boston, Inc., Boston, MA, 1993. MR1227098 (94m:17016)

[MZ] Ian M. Musson and Yi Ming Zou, Crystal bases for $U_{q}(\operatorname{osp}(1,2 r))$, J. Algebra 210 (1998), no. 2, 514-534, DOI 10.1006/jabr.1998.7591. MR.1662280(99j:17024) 
[Wa] Weiqiang Wang, Double affine Hecke algebras for the spin symmetric group, Math. Res. Lett. 16 (2009), no. 6, 1071-1085, DOI 10.4310/MRL.2009.v16.n6.a14. MR2576694 (2011a:20009)

[Ya] Hiroyuki Yamane, Quantized enveloping algebras associated with simple Lie superalgebras and their universal R-matrices, Publ. Res. Inst. Math. Sci. 30 (1994), no. 1, 15-87, DOI 10.2977/prims/1195166275. MR 1266383 (95d:17017)

[Zou] Yi Ming Zou, Integrable representations of $U_{q}(\operatorname{osp}(1,2 n))$, J. Pure Appl. Algebra 130 (1998), no. 1, 99-112, DOI 10.1016/S0022-4049(97)00088-1. MR1632799 (99e:17028)

Department of Mathematics, 567 Lake Hall, Northeastern University, Boston, MassachusetTs 02115

E-mail address: se.clark@neu.ed

Department of Mathematics, University of Virginia, Charlottesville, Virginia 22904

E-mail address: deh4n@virginia.edu

Department of Mathematics, University of Virginia, Charlottesville, Virginia 22904

E-mail address: ww9c@virginia.edu 